\title{
Probing isovector scalar mesons in the charmless three-body $B$ decays
}

\author{
Jian Chai $\odot,{ }^{1}$ Shan Cheng $\odot,{ }^{1,2,}$ and Ai-Jun $\mathrm{Ma}^{3}$ \\ ${ }^{1}$ School of Physics and Electronics, Hunan University, Changsha 410082, China \\ ${ }^{2}$ School for Theoretical Physics, Hunan University, Changsha 410082, China \\ ${ }^{3}$ Department of Mathematics and Physics, Nanjing Institute of Technology, Nanjing 211167, China
}

(Received 18 October 2021; accepted 27 January 2022; published 17 February 2022)

\begin{abstract}
We propose to study the multiparticle configurations of isovector scalar mesons, $a_{0}(980)$ and $a_{0}(1450)$, in the charmless three-body $B$ decays by considering the width effects. Two scenarios of $a_{0}$ configurations are assumed in which the first one takes $a_{0}(980)$ as the lowest-lying $q \bar{q}$ state and $a_{0}(1450)$ as the first radial excited state, the second one takes $a_{0}(1450)$ as the lowest-lying $q \bar{q}$ state and $a_{0}(1950)$ as the first radial excited state while $a_{0}(980)$ is not a $q \bar{q}$ state. Within these two scenarios, we do the perturbative QCD (PQCD) calculation for the quasi-two-body $B \rightarrow a_{0}[\rightarrow K \bar{K} / \pi \eta] h$ decays and extract the corresponding branching fractions of two-body $B \rightarrow a_{0} h$ decays under the narrow width approximation. Our predictions show that the first scenario of the $a_{0}(980)$ configuration cannot be excluded by the available measurements in $B$ decays, and the contributions from $a_{0}(1450)$ to the branching fractions in most channels are comparable in the first and second scenarios. Several channels are suggested for the forthcoming experimental measurements to reveal the multiparticle configurations of $a_{0}$, such as the channel $B^{0} \rightarrow a_{0}^{-}(980)\left[\rightarrow \pi^{-} \eta\right] \pi^{+}$with the largest predicted branching fraction, the channels $B^{0} \rightarrow$ $a_{0}^{ \pm}(1450)\left[\rightarrow K^{ \pm} \bar{K}^{0}, \pi^{ \pm} \eta\right] \pi^{\mp}$ whose branching fractions obtained in the second scenario is about three times larger in magnitude than that obtained in the first scenario, and also the channels $B^{+} \rightarrow$ $a_{0}^{+}(1950)\left[K^{+} \bar{K}^{0} / \pi^{+} \eta\right] K^{0}$ whose branching fractions are linearly dependent on the partial width $\Gamma_{a_{0}(1950) \rightarrow K K / \pi \eta}$.
\end{abstract}

DOI: 10.1103/PhysRevD.105.033003

\section{INTRODUCTION}

It is known that the scalar mesons with the masses below and near $1 \mathrm{GeV}$, say the isoscalar mesons $\sigma / f_{0}(500)$ and $f_{0}(980)$, the isovector $a_{0}(980)$ and the isodoublet $\kappa$, form a $S U$ (3) flavor nonet. Meanwhile, the mesons heavier than $1 \mathrm{GeV}$, including $f_{0}(1370), f_{0}(1500), a_{0}(1450)$, and $K_{0}^{*}(1430)$, make up another nonet. The underlying assignment of the heavier nonet is almost accepted as the quarkantiquark configuration replenished with some possible gluon content [1-4], while the inner nature of scalar mesons in the lighter nonet is still not clear [5-7], even though the compact tetraquark state [8-10] and the $K \bar{K}$ bound state [11] are the most favorite two candidates nowadays. This is easy to understand from the views of spectral analysis at low energy because the scalar meson in $q \bar{q}$ configuration has a unit of orbital angular momentum which increases their masses. In contrast, it is not necessary

\footnotetext{
*scheng@hnu.edu.cn
}

Published by the American Physical Society under the terms of the Creative Commons Attribution 4.0 International license. Further distribution of this work must maintain attribution to the author(s) and the published article's title, journal citation, and DOI. Funded by SCOAP. to introduce the orbital angular momentum when the scalar meson is in the $q^{2} \bar{q}^{2}$ configuration [12]. The case becomes different in the weak decays like $B \rightarrow$ $f_{0}(980) l \nu$ with large recoiling, where the conventional $q \bar{q}$ assignment can be expected to be dominated in the energetic $f_{0}(980)$ since the possibility to form a tetraquark state is power suppressed compared to the state of the quark pair [13]; meanwhile, the final state interaction (FSI) is weak too. However, this argument encounters a challenge from the PQCD calculation of $B \rightarrow a_{0}(980) K$ decays [14], where the theoretical predictions of branching fractions are much larger than that of the measured upper limits. We would like to comment that their calculation is carried out in the static $a_{0}(980)$ approximation while the experiment measurement is actually fulfilled by the $\pi \eta$ invariant mass spectral. It is apparent that the salient property of scalar mesons, say, the large decay width which cause a strong overlap between resonances and background, and subsequently influence the PQCD prediction.

The width effect of intermediate resonant states has been studied in three-body $B$ decays with a large number of channels by variable theoretical approaches based on QCD, due to the significant physics to understand the hadron structures and also the matter-antimatter asymmetry. 
We here highlight some developments in this research filed in the order of different theoretical approaches.

$P Q C D$ A global analysis of three-body charmless decays in the type of $B \rightarrow V\left[\rightarrow P_{1} P_{2}\right] P_{3}{ }^{1}$ is performed [15] to determine the lowest several Gegenbauer moments of two-meson system, which are the nonperturbative inputs describing the nonasymptotic QCD correction effect in the light-cone distribution amplitudes (LCDAs). In Ref. [16], the factorization formulas of PQCD is expanded in the four-body $B$ decay to two $[K \pi]_{S, P}$ pairs with the invariant mass around the $K^{*}(892)$ resonance, some further observations like the triple-product asymmetries and the $S$-wave induced direct $C P$ asymmetries are presented with the interference between different helicity amplitudes. Motivated by the measurement of significant derivations from the simple phase-space model in the channels $B \rightarrow K \bar{K} P_{1}$ and $B_{(s)} \rightarrow D_{(s)} P_{1} P_{2}$ at $\mathrm{B}$ factories and LHC, the virtual contribution clarified by the experiment collaborations is understood theoretically by the Breit-Wigner-tail (BWT) effects from the corresponding intermediate resonant states, say $\rho, \omega$ and $D_{(s)}^{*}$, respectively $[17,18]$.

$Q C D F$ The QCD factorization (QCDF) formula of amplitudes in three-body $B$ decays [19] is parametrized in a new way where the contributions from valence $u$ and $c$ quark are separated, and a new source of $C P$ violation can be generated via the strong phase with the opening of $D \bar{D}$ threshold in the high invariant mass region [20]. Motivated by the next-to-next-toleading-order $\alpha_{s}\left(m_{b}\right)$ correction and the finite width effect, three-body $B$ decay is studied from the point of view of factorization for the heavy-to-heavy $B \rightarrow D \rho[\rightarrow \pi \pi], D K^{*}[\rightarrow K \pi]$ decays in the kinematics with small invariant mass of dimeson system [21]. Very recently, a novel observation named the forwardbackward asymmetry induced $C P$ asymmetry (FBI$C P A)$ is introduced in the three-body heavy meson decays, the estimation based on the generalized factorization approach implies that the FBI- CPA in the channel $D^{ \pm} \rightarrow K^{+} K^{-} \pi^{ \pm}$is about a milli, which is at the same order of current experiment measurement capability [22]. In Refs. [23,24], the finite-width effects of intermediate resonant states in three-body $B / D$ decays is expressed by a correlation parameter $\eta_{R}$ and the evaluation is carried out in QCDF.

LCSRs The width effect of intermediate resonant $\rho$ and its radial excited states is discussed in detail in the $P$-wave $B \rightarrow \pi \pi$ transition form factors from the $B$ meson light-cone sum rules (LCSRs) approach [25],

\footnotetext{
${ }^{1}$ Here $V, P$ denote the vector and pseudoscalar meson, respectively, and $S$ indicates the scalar meson in the following. In the fit, only the $P_{1} P_{2}=\pi \pi, \pi K, K \bar{K}$ channels are considered due to the experiment precision.
}

revealing the sizeable effects from width and background $(20 \%-30 \%)$ to the conventional treatment in the single narrow-width approximation for the LCSRs prediction of the $B \rightarrow \rho$ transition form factors. This result is confirmed by the other independent LCSRs with dipion distribution amplitudes (DAs) where the hadronic dipion state has a small invariant mass and simultaneously a large recoil [26,27]. The further studies are carried out for the $P$-wave $B \rightarrow K \pi$ form factors with the isodouble intermediate resonances $K_{0}^{*}$ [28] and the $B_{s} \rightarrow K \bar{K}$ form factors with the isoscalar scalar intermediate resonances $f_{0}(980)$ and $f_{0}(1450)$ [13].

The above considerations mainly focus on the $P$-wave and isoscalar $S$-wave contributions from the intermediate resonant states, while the study of isovector scalar intermediate resonance is still missing. In this paper we will demonstrate this issue in the framework of the PQCD approach. The motivations of this study are twofold. First, we perform the PQCD prediction of $B \rightarrow a_{0}(980)[\rightarrow \eta \pi] K$ decays that go beyond the single pole approximation, trying to explain the measurement status. Second, we consider the roles of $a_{0}(1450)$ and $a_{0}(1950)$ in the $B \rightarrow$ $\bar{K} K K$ decays inspired by the recent measurements of charm meson decays where $a_{0}(1450)$ and $a_{0}(1950)$ are observed in the $K \bar{K}$ invariant mass spectral [29-31], supplementing to the $B \rightarrow \eta \pi K$ decays observed first at the Crystal Barrel Collaboration a long time ago $[32,33]$. The study would be executed in parallel by taking two different scenarios of $a_{0}$ states, where the first one says that $a_{0}(980)$ is the lowest lying $q \bar{q}$ state and $a_{0}(1450)$ is the first excited state, and the second one states that $a_{0}(1450)$ and $a_{0}(1950)$ are the lowest lying $q \bar{q}$ state and the first excited state, respectively. Our calculations show that the $q \bar{q}$ configuration of $a_{0}(980)$ is not to be excluded by the available measurements in $B$ decays, which confirms the statements we made above. Predictions in this work would help us to probe the inner structure of energetic isovector scalar mesons. For example, (a) the channel $B^{0} \rightarrow a_{0}^{-}(980)\left[\rightarrow \pi^{-} \eta\right] \pi^{+}$has the largest branching fraction under the $q \bar{q}$ configuration of $a_{0}(980)$, (b) the branching fractions of channels $B^{0} \rightarrow$ $a_{0}^{ \pm}(1450)\left[\rightarrow K^{ \pm} \bar{K}^{0}, \pi^{ \pm} \eta\right] \pi^{\mp}$ obtained in the second scenario are about three times larger in magnitude than that obtained in the first scenario, even though the results obtained from two scenarios are close to each other in the most channels with the intermediate state $a_{0}(1450)$, (c) the branching fractions of channels $B^{+} \rightarrow$ $a_{0}^{+}(1950)\left[K^{+} \bar{K}^{0} / \pi^{+} \eta\right] K^{0}$ are linear dependent on the partial width $\Gamma_{a_{0}(1950) \rightarrow K K / \pi \eta}$ in the second scenario.

This article is organized as follows. In Sec. II, the framework of the PQCD approach to deal with the resonance contribution in three-body $B$ decays is briefly described in terms of kinematics and dynamics. Section III presents the PQCD predictions of the $B \rightarrow a_{0}[\rightarrow K \bar{K}, \eta \pi] h$ 
decays with some discussions. We summarize in Sec. IV. The PQCD predictions on $B_{s}$ decays are presented in the Appendix $A$, and the factorization formulas of the related quasi-two-body decay amplitudes are listed in Appendix B.

\section{KINEMATICS AND DYNAMICS}

Concerning three-body $B$ decays, there are three typical kinematical configurations in the physical Dalitz plot of two independent invariant masses by considering the fourmomentum conservation, in which only the kinematical region with collinear decay products can be calculated reliably from the perturbative theory based on the factorization hypothesis [18]. The other two kinematical regions with the three energetic decay products and a soft decay product configurations are either lacking the rigorous factorization proof or are beyond the available perturbative picture of heavy meson decays. Collinear decay products means that two energetic hadrons move ahead with collinear momenta while the rest recoil back, ${ }^{2}$ corresponding to the intermediate parts of three edges in the Dalitz plot.

The matrix element from vacuum to collinear two meson system sandwiched with certain two quark operators is defined by the dimeson DAs, the chirally even two quark dimeson DA is quoted for example as [34]

$$
\begin{aligned}
& \left\langle M_{1}^{a}\left(p_{1}\right) M_{2}^{b}\left(p_{2}\right)\left|\bar{q}_{f}(x n) \tau q_{f^{\prime}}(0)\right| 0\right\rangle \\
& \quad=\kappa_{a b} \int d z e^{i z x\left(p_{R} \cdot n\right)} \Phi_{M_{1} M_{2}}^{a b, f f^{\prime}}(z, \zeta, s),
\end{aligned}
$$

where the indexes $f, f^{\prime}$ respect the (anti)quark flavor; $a, b$ indicate the electric charge of each meson, $\kappa_{a b}$ is the isospin symmetry coefficient which in the case of dipion system reads $\kappa_{+-/ 00}=1$ and $\kappa_{+0}=\sqrt{2}, p_{R}=k_{1}+k_{2}$ is the total momentum of dimeson state, $\tau=1 / 2, \tau_{3} / 2$ correspond to the isoscalar and isovector dimeson DAs, respectively. The generalized dimeson DA $\Phi_{\|}^{a b, f f^{\prime}}$ is characterized by three independent kinematical variables, say the momentum fraction $z$ carried by the antiquark, the longitudinal momentum fraction carried by one of the mesons $\zeta=$ $p_{1}^{+} / p_{R}^{+}$and the invariant mass squared $s=p_{R}^{2}$. Besides the conventional Gegenbauer expansion stemmed from the eigenfunction of QCD evolution equation, the partial wave expansion considered in the dimeson system contributes the other Legendre polynomial $C_{l}^{1 / 2}$. The double expansion of two quark dimeson DA is written as

$$
\begin{aligned}
\Phi_{M_{1} M_{2}}^{I=1}(z, \zeta, s, \mu)= & 6 z(1-z) \sum_{n=0, \text { even }}^{\infty} \sum_{l=1, \text { odd }}^{n+1} B_{n l}^{I=1}(s, \mu) \\
& \times C_{n}^{3 / 2}(2 z-1) C_{l}^{1 / 2}(2 \zeta-1),
\end{aligned}
$$

\footnotetext{
${ }^{2} E_{i} \sim m_{B} / 2$ and $E_{j}+E_{k} \sim m_{B} / 2$ in the massless approximation of final mesons.
}

here the even Gegenbauer index $n$ and the odd partial-wave index $l$ are guaranteed by the $C$ parity. For the expansion coefficients $B_{n l}$, they have a similar scale dependence as the Gegenbauer moments of single pion and rho mesons. In the narrow width approximation in the vicinity of the resonance, dimenson DAs reduce to the DAs of the relative resonance, indicating that the Gegenbuer moments of the intermediate resonance are actually proportional to the expansion coefficient at zero point with the lowest partial wave, says $a_{n}^{R}(\mu) \propto B_{n 1}(s=0, \mu)$. In this way, the decay constant of intermediate resonance is proportional to the product of its decay width with the imaginary part of first expansion coefficient at the resonant pole, that is $f_{R} \propto$ $\Gamma_{R} \operatorname{Im}\left[B_{01}\left(m_{R}^{2}\right)\right][35]$.

With this definition, the dimeson DAs are the most general objects to describe the dimeson mass spectrum in hard production processes whose asymptotic formula indicates the information of the deviation from the unstable intermediate resonant meson DAs. After integrating over the momentum fraction of antiquark, the isovector scalar dimeson DA in our interest is normalized to the timelike meson form factor as

$$
\int_{0}^{1} d z \Phi_{M_{1} M_{2}}^{I=1}(z, \zeta, s)=(2 \zeta-1) \Gamma_{M_{1} M_{2}}^{I=1}(s)
$$

where the timelike form factor at zero energy point is normalized to unit as $\Gamma_{M_{1} M_{2}}^{I=1}(0)=1$. When the invariant mass of dimeson system is small, the higher $\mathcal{O}(s)$ terms in the expansion of coefficient $B_{n l}(s, \mu)$ around the resonance pole can be safely neglected due to the large suppression $\mathcal{O}\left(s / m_{b}^{2}\right)$ in contrast to the energetic dimeson system in $B$ decay, so the relation $B_{n 1}(s, \mu) \rightarrow a_{n}(\mu) \Gamma_{M_{1} M_{2}}^{I=1}(s)$ can be obtained in the lowest partial wave approximation. This argument induces the basic assumption in PQCD that the energetic dimeson DAs can be deduced from the DAs of resonant meson by replacing the decay constant by the timelike form factor.

The isovector scalar form factor of $K \bar{K}$ and $\pi \eta$ systems are defined by the local matrix elements sandwiched by two quark operator $[36,37]$

$$
\begin{aligned}
\left\langle K^{-} K^{0}\left(\pi^{-} \eta\right)\left|\bar{u}(0) \frac{\tau_{3}}{2} d(0)\right| 0\right\rangle & =\frac{m_{\pi}^{2}}{m_{u}+m_{d}} \Gamma_{K \bar{K}(\pi \eta)}^{I=1}(s) \\
& \equiv B_{0} \Gamma_{K \bar{K}(\pi \eta)}^{I=1}(s)
\end{aligned}
$$

with the normalization conditions $\Gamma_{K \bar{K}}^{I=1}(0)=1$ and $\Gamma_{\pi \eta}^{I=1}(0)=\sqrt{6} / 3$. In the single resonance approximation, we insert a $a_{0}$ state in the matrix elements 


$$
\begin{aligned}
& \left\langle K^{-} K^{0}\left(\pi^{+} \eta\right)\left|\bar{u}(0) \frac{\tau_{3}}{2} d(0)\right| 0\right\rangle \\
& \approx \frac{\left\langle K^{-} K^{0}\left(\pi^{+} \eta\right) \mid a_{0}^{-}\right\rangle\left\langle a_{0}^{-}\left|\bar{u}(0) \frac{\tau_{3}}{2} d(0)\right| 0\right\rangle}{\mathcal{D}_{a_{0}}} \\
& =\frac{g_{a_{0} K \bar{K}(\pi \eta)} m_{a_{0}} \bar{f}_{a_{0}}}{\mathcal{D}_{a_{0}}},
\end{aligned}
$$

and ultimately arrive at

$$
\Gamma_{K \bar{K}(\pi \eta)}^{I=1}(s)=\frac{g_{a_{0} K \bar{K}(\pi \eta)} m_{a_{0}} \bar{f}_{a_{0}}}{B_{0} \mathcal{D}_{a_{0}}} .
$$

Several comments are supplied to demonstrate this expression.

(i) The decay constants of scalar meson are defined with the scalar and vector currents,

$$
\begin{aligned}
\left\langle S\left|\bar{u}(0) \frac{\tau_{3}}{2} d(0)\right| 0\right\rangle & =m_{S} \bar{f}_{S}, \\
\left\langle S(p)\left|\bar{u}(0) \gamma_{\mu} \frac{\tau_{3}}{2} d(0)\right| 0\right\rangle & =p_{\mu} f_{S} .
\end{aligned}
$$

They are related by the equations of motion $\frac{m_{S} f_{S}}{m_{u}-m_{d}}=\bar{f}_{S}(\mu)$, indicating that the neutral scalar meson cannot be produced via the vector current because of the charge conjugation invariance or the conservation of vector current, but the constant $\bar{f}_{S}$ is still finite.

(ii) Under the narrow $a_{0}$ approximation, the matrix element of strong decay is defined by the coupling [38]

$$
\left\langle K^{-} K^{0}\left(\pi^{+} \eta\right) \mid a_{0}^{-}\right\rangle=g_{a_{0} K \bar{K}(\pi \eta)}=\sqrt{\frac{8 \pi m_{a_{0}}^{2} \Gamma_{a_{0} \rightarrow K \bar{K}(\pi \eta)}}{q_{0}}}
$$

with the energy independent partial decay width ${ }^{3}$ $\Gamma_{a_{0} \rightarrow K \bar{K}(\pi \eta)}$. In the definition, $q_{0}=q\left(m_{a_{0}}^{2}\right)$ is the magnitude of daughter meson $[K(\pi)$ or $\bar{K}(\eta)]$ momentum

$$
\begin{aligned}
& q(s) \\
& =\frac{1}{2} \sqrt{\left[s-\left(m_{K(\pi)}+m_{\bar{K}(\eta)}\right)^{2}\right]\left[s-\left(m_{K(\pi)}-m_{\bar{K}(\eta)}\right)^{2}\right] / s}
\end{aligned}
$$

at $a_{0}$ mass. We take the renormalized mass of $a_{0}$ rather than the pole mass obtained from $T$-matrix analysis, since the mass and width parameter are

\footnotetext{
${ }^{3}$ The partial widths of $a_{0}^{0} \rightarrow K \bar{K}$ decays have the relations $\Gamma_{a_{0}^{0} \rightarrow K^{+} K^{-}}=\Gamma_{a_{0}^{0} \rightarrow K^{0} \bar{K}^{0}}=\Gamma_{a_{0} \rightarrow K \bar{K}} / 2$.
}

strongly distorted with lying just below the opening of the $K \bar{K}$ channel and hence generating an important cusplike behavior in the resonant amplitude [39]. Actually, $q=\sqrt{s} \beta(s)$ with $\beta(s)$ being the nondimensional phase space factor of $K \bar{K}(\pi \eta)$ system, which reflects the information of momentum difference described by the variable $\zeta$ mentioned in the dimeson DAs.

(iii) We take the conventional energy-dependent BreitWigner denominator for $a_{0}^{\prime}$ and $a_{0}^{\prime \prime}$ mesons, ${ }^{4}$

$$
\mathcal{D}_{a_{0}^{\prime}}=m_{a_{0}^{\prime}}^{2}-s-i m_{a_{0}^{\prime}} \Gamma_{a_{0}^{\prime}}^{\mathrm{tot}} \frac{q(s)}{q_{0}} \frac{m_{a_{0}^{\prime}}}{\sqrt{s}},
$$

where $\Gamma_{a_{0}^{\prime}}^{\text {tot }}$ is the total decay widths of resonant state meson $a_{0}^{\prime}$. For the meson $a_{0}(980)$, we consider the Flatté model [40]

$$
\mathcal{D}_{a_{0}}=m_{a_{0}}^{2}-s-i\left(g_{\pi \eta}^{2} \beta_{\pi \eta}+g_{K \bar{K}}^{2} \beta_{K \bar{K}}\right),
$$

the coupling constants $g_{\pi \eta}=0.324 \mathrm{GeV}$ and $g_{K \bar{K}}^{2} / g_{\pi \eta}^{2}=1.03$ are fixed by the isobar model fits [39]. Furthermore, we can get $g_{a_{0} \pi \eta}=2.297 \mathrm{GeV}$ and $g_{a_{0} K \bar{K}}=2.331 \mathrm{GeV}$ with the relations $g_{K \bar{K}}=$ $g_{a_{0} K \bar{K}} /(4 \sqrt{\pi})$ and $g_{\pi \eta}=g_{a_{0} \pi \eta} /(4 \sqrt{\pi})$. We mark that, in the $a_{0} \rightarrow \pi \eta$ channel, the phase factor $\beta_{K \bar{K}}$ could also be pure imaginary number when the invariant mass of $\pi \eta$ state is small than the threshold value of $K \bar{K}$ state, the contribution from this region interacts destructively with that from the rest region of $\pi \eta$ invariant mass.

With rearranging the kinematical variable $\zeta$ into the daughter meson momentum $q(s)$ and considering the $S U$ (3) symmetry, the matrix element from vacuum to $\mathrm{S}$ wave $K \bar{K} / \pi \eta$ state can be decomposed as [12]

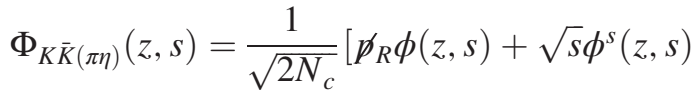

$$
\begin{aligned}
& \left.+\sqrt{s}(\not \not h-1) \phi^{t}(z, s)\right] \text {. }
\end{aligned}
$$

In the lowest partial-wave accuracy, the twist 2 LCDA is written as [41]

$$
\begin{aligned}
\phi(z, s)= & \frac{\Gamma_{K \bar{K}(\pi \eta)}(s)}{2 \sqrt{2 N_{c}}} 6 z(1-z) \\
& \times\left[\frac{f_{S}}{\bar{f}_{S}(\mu)}+\sum_{m=1}^{\infty} B_{m}(\mu) C_{m}^{3 / 2}(2 z-1)\right],
\end{aligned}
$$

with $B_{0}(\mu) \equiv f_{S} / \bar{f}_{S}(\mu) \gg 1$. It is clear that the even Gegenbauer coefficients $B_{m}$ are suppressed and the odd

\footnotetext{
${ }^{4}$ Hereafter we use the abbreviations $a_{0}, a_{0}^{\prime}$ and $a_{0}^{\prime \prime}$ to denote $a_{0}(980), a_{0}(1450)$, and $a_{0}(1950)$, respectively.
} 


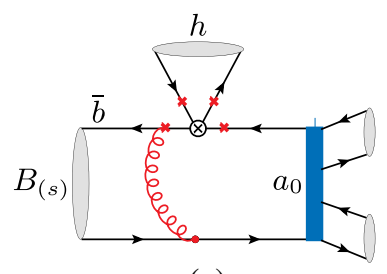

(a)

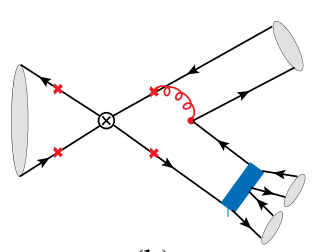

(b)

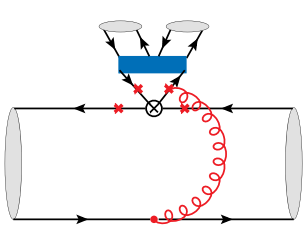

(c)

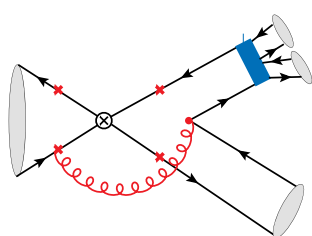

(d)

FIG. 1. Typical Feynman diagrams of the $B_{(s)} \rightarrow a_{0}[\rightarrow K \bar{K} / \pi \eta] h$ decays with $h=\pi, K$ in the PQCD approach.

Gegenabauer moments are dominated in the twist 2 LCDA of scalar meson, this is definitely different from the $\pi$ and $\rho$ mesons in which the odd moments vanish. The twist 3 LCDAs are

$\begin{aligned} \phi^{s}(z, s) & =\frac{\Gamma_{K \bar{K}(\pi \eta)}(s)}{2 \sqrt{2 N_{c}}}\left[1+\sum_{m=1}^{\infty} a_{m}(\mu) C_{m}^{1 / 2}(2 z-1)\right], \\ \phi^{t}(z, s) & =\frac{\Gamma_{K \bar{K}(\pi \eta)}(s)}{2 \sqrt{2 N_{c}}}(1-2 z)\left[1+\sum_{m=1}^{\infty} b_{m}(\mu) C_{m}^{1 / 2}(2 z-1)\right] .\end{aligned}$

The definitions of $B$ meson and light meson wave functions and the models of their LCDAs, as well as the basic procedures of PQCD approach to deal with the so called quasi-two-body $B$ decays as a marriage problem, can be found in detail in Ref. [18].

In Fig. 1, we depict the typical Feynman diagrams of the $B \rightarrow a_{0}[\rightarrow K \bar{K} / \pi \eta] h$ decays with $h=\pi, K$ in the PQCD approach, in which the symbols $\otimes$ and $\times$ denote the vertex of weak interaction and the possible attachments of hard gluons, respectively, the rectangle indicates the intermediate resonant states $a_{0}$ and the subsequent strong decays $a_{0} \rightarrow K \bar{K} / \pi \eta$. In the $B$ meson rest frame, the explicit definitions of kinematics in the $B\left(p_{B}\right) \rightarrow R\left(p_{R}\right)\left[\rightarrow h_{1}\left(p_{1}\right) h_{2}\left(p_{2}\right)\right] h_{3}\left(p_{3}\right)$ decays are considered as follows,

$p_{B}=\frac{m_{B}}{\sqrt{2}}(1,1, \mathbf{0}), \quad k_{B}=\left(0, \frac{m_{B}}{\sqrt{2}} x_{B}, \mathbf{k}_{\mathbf{B T}}\right)$,

$p_{R}=\frac{m_{B}}{\sqrt{2}}(1, \xi, \mathbf{0}), \quad k_{R}=\left(\frac{m_{B}}{\sqrt{2}} z, 0, \mathbf{k}_{\mathbf{T}}\right)$,

$p_{3}=\frac{m_{B}}{\sqrt{2}}(0,1-\xi, \mathbf{0}), \quad k_{3}=\left(0, \frac{m_{B}}{\sqrt{2}}(1-\xi) x_{3}, \mathbf{k}_{\mathbf{3}}\right)$,

where $k_{B}, k_{R}$, and $k_{3}$ are the momenta carried by the antiquark in the meson states with the momentum fractions $x_{B}, z$ and $x_{3}$, respectively. The new variable $\xi \equiv s / m_{B}^{2}$ indicates the momentum transfer from $B$ meson to resonant state $R$. The differential branching ratios for the quasi-twobody $B_{(s)} \rightarrow a_{0}[\rightarrow K \bar{K} / \pi \eta] h$ decays is written as [42]

$$
\frac{d \mathcal{B}}{d \zeta}=\frac{\tau_{B} q_{h}(s) q(s)}{64 \pi^{3} m_{B_{(s)}}} \overline{|\mathcal{A}|^{2}},
$$

in which daughter meson momentum $q(s)$ has been defined in Eq. (9), and $q_{h}(s)$ is the magnitude of momentum for the bachelor meson $h$

$q_{h}(s)=\frac{1}{2} \sqrt{\left[\left(m_{B}^{2}-m_{h}^{2}\right)^{2}-2\left(m_{B}^{2}+m_{h}^{2}\right) s+s^{2}\right] / s}$.

The decaying amplitudes is exactly written as a convolution of the hard kernel $H$ with the hadron distribution amplitudes (DAs) $\phi_{B}, \phi_{h}$ and $\phi_{K \bar{K}, \pi \eta}$

$$
\begin{aligned}
& \mathcal{A}\left(B_{(s)} \rightarrow a_{0}[\rightarrow K \bar{K} / \pi \eta] h\right) \equiv\left\langle[K \bar{K} / \pi \eta]_{a_{0}} h\left|\mathcal{H}_{\mathrm{eff}}\right| B_{(s)}\right\rangle \\
& =\phi_{B}\left(x_{1}, b_{1}, \mu\right) \otimes H\left(x_{i}, b_{i}, \mu\right) \otimes \phi_{K \bar{K} / \pi \eta}(x, b, \mu) \\
& \quad \otimes \phi_{h}\left(x_{3}, b_{3}, \mu\right),
\end{aligned}
$$

in which $[K \bar{K} / \pi \eta]_{a_{0}}$ indicates the dimeson system in our interesting, $\mu$ is the factorization scale, $b_{i}$ are the conjugate distances of transversal momenta. We present the expressions of amplitudes $\mathcal{A}$ for the considered decaying processes in the Appendix B. Under the narrow width approximation

$$
\begin{aligned}
\mathcal{A} & =\int d s \frac{\left\langle K \bar{K} / \pi \eta \mid a_{0}\right\rangle\left\langle a_{0} h\left|\mathcal{H}_{\mathrm{eff}}\right| B_{(s)}\right\rangle}{\left[m_{a_{0}}^{2}-s-i m_{a_{0}} \Gamma_{a_{0}}(s)\right]} \\
& \rightarrow\left\langle K \bar{K} / \pi \eta \mid a_{0}\right\rangle\left\langle a_{0} h\left|\mathcal{H}_{\mathrm{eff}}\right| B_{(s)}\right\rangle,
\end{aligned}
$$

we can extract the branching fractions of two-body decays from the quasi-two-body decays by

$$
\begin{aligned}
& \mathcal{B}\left(B_{(s)} \rightarrow a_{0}[\rightarrow K \bar{K} / \pi \eta] h\right) \\
& \quad \approx \mathcal{B}\left(B_{(s)} \rightarrow a_{0} h\right) \cdot \mathcal{B}\left(a_{0} \rightarrow K \bar{K} / \pi \eta\right) .
\end{aligned}
$$

\section{NUMERICS AND DISCUSSIONS}

In Table I, we present the PDG averaged value for the masses and total widths of single mesons, as well as the Wolfenstein parameters of CKM matrix. The $B_{(s)}$ meson wave function relies on the three independent parameters, the mass $m_{B}$, the decay constant $f_{B}$ and the first inverse moment $\omega_{B}$. For the inverse moment $\omega_{B}$, we take the interval $\omega_{B}(1 \mathrm{GeV})=0.40 \pm 0.04 \mathrm{GeV}$ and $\omega_{B_{s}}(1 \mathrm{GeV})=$ $0.50 \pm 0.05 \mathrm{GeV}$ obtained by the QCD sum rules [43] with considering smaller uncertainty. The mean lives of $B$ 
mesons are also taken from PDG, they are $\tau_{B^{ \pm}}=$ $1.638 \times 10^{-12} \mathrm{~s}, \quad \tau_{B^{0}}=1.520 \times 10^{-12} \mathrm{~s}, \quad$ and $\quad \tau_{B_{s}}=$ $1.509 \times 10^{-12} \mathrm{~s}$.

The PDG value of light meson decay constant follows from the lattice QCD average $f_{K^{+}} / f_{\pi^{+}}=1.193$ [44]. We truncate to the second moments for the Gegenbauer expansion of leading twist LCDAs, and take $a_{1}^{\pi}=0$ and $a_{2}^{\pi}(1 \mathrm{GeV})=0.270 \pm 0.047$ obtained recently from the LCSR fit [45] of the pion electromagnetic form factor. ${ }^{5}$ For the kaon meson, we take the lattice result obtained by using $N_{f}=2+1$ sea quarks and the domain-wall fermions [52], say, $a_{1}^{K}(1 \mathrm{GeV})=0.060 \pm 0.004$ and $a_{2}^{K}(1 \mathrm{GeV})=$ $0.175 \pm 0.065$, which is comparable with the QCD sum rules calculations [50,53] and the result from DysonSchwinger equations with dynamical chiral spontaneously breaking (DCSB)-improved kernel [54]. We take the chiral masses at $m_{0}^{\pi}=1.913 \mathrm{GeV}, m_{0}^{K}=1.892 \mathrm{GeV}$ with considering the well-known chiral perturbative theory $(\chi \mathrm{PT})$ relations [55]

$$
m_{0}^{\pi}=\frac{m_{\pi}^{2} \mathcal{R}}{2 m_{s}}, \quad m_{0}^{K}=\frac{m_{K}^{2}}{m_{s}\left[1+\frac{1}{\mathcal{R}}\left(1-\frac{\mathcal{R}^{2}-1}{4 \mathcal{Q}^{2}}\right)\right]},
$$

in which $\mathcal{R} \equiv 2 m_{s} /\left(m_{u}+m_{d}\right)=24.4 \pm 1.5, \mathcal{Q}^{2} \equiv\left[m_{s}^{2}-\right.$ $\left.\left(m_{u}+m_{d}\right)^{2} / 4\right] /\left(m_{d}^{2}-m_{u}^{2}\right)=(22.7 \pm 0.8)^{2}$, the current quark masses are $\bar{m}_{s}(1 \mathrm{GeV})=0.125 \mathrm{GeV}, \bar{m}_{d}(1 \mathrm{GeV})=$ $0.0065 \mathrm{GeV}$, and $\bar{m}_{u}(1 \mathrm{GeV})=0.0035 \mathrm{GeV}$. For the twist 3 LCDA, we only take into account the asymptotic terms in the numerical analysis.

Concerning the intermediate resonant isovector scalar states $a_{0} \mathrm{~s}$, the main inputs are the timelike form factor entered in each LCDA and the Gegenbauer moments in the leading twist LCDA. To reveal the timelike form factor described in Eq. (6), we use the QCD sum rules predictions on the decay constants [12], they are $\bar{f}_{a_{0}}(1 \mathrm{GeV})=$ $0.365 \pm 0.020 \mathrm{GeV}$ and $\bar{f}_{a_{0}^{\prime}}(1 \mathrm{GeV})=-0.280 \pm 0.035 \mathrm{GeV}$ obtained in the first scenario where $a_{0}$ is treated as the lowest lying $q \bar{q}$ state and $a_{0}^{\prime}$ as the first excited state, and $\bar{f}_{a_{0}^{\prime}}(1 \mathrm{GeV})=0.460 \pm 0.050 \mathrm{GeV}$ and $\bar{f}_{a_{0}^{\prime \prime}}(1 \mathrm{GeV})=$ $0.390 \pm 0.040 \mathrm{GeV}$ obtained in the second scenario where $a_{0}^{\prime}$ is the lowest lying $q \bar{q}$ state and $a_{0}^{\prime \prime}$ as the first excited state. As shown in Eq. (8), the strong coupling constants $g_{a_{0} K \bar{K}}$ and $g_{a_{0} \pi \eta}$ are decided by the partial decay widths, which are fixed by the following considerations

(i) With the measurements $\left(\Gamma_{a_{0} \rightarrow \pi \eta} \times \Gamma_{a_{0} \rightarrow \gamma \gamma}\right) / \Gamma^{\text {tot }}=$ $0.21 \mathrm{keV}$ and $\Gamma_{a_{0} \rightarrow \gamma \gamma}=0.30 \pm 0.10 \mathrm{keV}$ [56], one gets $\Gamma_{a_{0} \rightarrow \pi \eta}=0.053 \pm 0.018 \mathrm{GeV}$. We do not use Eq. (8) to determine the partial width since it is an approximation expression under the narrow width limit. Furthermore, one can get $\Gamma_{a_{0} \rightarrow K \bar{K}}=0.009 \pm 0.003 \mathrm{GeV}$ with the measurement $\Gamma_{a_{0} \rightarrow K \bar{K}} / \Gamma_{a_{0} \rightarrow \pi \eta}=0.177$ [42].

(ii) The partial decay widths of $a_{0}^{\prime}$ to $K \bar{K}$ and $\pi \eta$ states are decided by the measured branching ratios $\Gamma_{a_{0}^{\prime} \rightarrow K \bar{K}} / \Gamma_{a_{0}^{\prime}}^{\mathrm{tot}}=0.082 \pm 0.028$ and $\Gamma_{a_{0}^{\prime} \rightarrow \pi \eta} / \Gamma_{a_{0}^{\prime}}^{\mathrm{tot}}=$ $0.093 \pm 0.020$ [42].

(iii) For the $a_{0}^{\prime \prime}$ decays, there is no direct measurement and the predictions from different models vary widely. For example, the extended linear sigma model (eLSM) states that $\Gamma_{a_{0}^{\prime \prime}} \rightarrow K \bar{K}=94 \pm$ $54 \mathrm{MeV}$ and $\Gamma_{a_{0}^{\prime \prime}} \rightarrow \pi \eta=94 \pm 16 \mathrm{MeV}$ [57], while the $3^{3} P_{0}$ quark model gives the result $0.74 \mathrm{MeV}$ and $5.13 \mathrm{MeV}$ correspondingly [58]. So in our evaluation, we take the largest interval of this variable to account its uncertainty.

(iv) To close the descriptions, we summarize the intervals of partial decay widths as

$$
\begin{array}{ll}
\Gamma_{a_{0} \rightarrow K \bar{K}}=0.009 \pm 0.003 \mathrm{GeV}, & \Gamma_{a_{0} \rightarrow \pi \eta}=0.053 \pm 0.018 \mathrm{GeV}, \\
\Gamma_{a_{0}^{\prime} \rightarrow K \bar{K}}=0.022 \pm 0.008 \mathrm{GeV}, & \Gamma_{a_{0}^{\prime} \rightarrow \pi \eta}=0.025 \pm 0.006 \mathrm{GeV}, \\
\Gamma_{a_{0}^{\prime \prime} \rightarrow K \bar{K}} \in[0,0.150] \mathrm{GeV}, & \Gamma_{a_{0}^{\prime \prime} \rightarrow \pi \eta} \in[0,0.110] \mathrm{GeV} .
\end{array}
$$

Concerning the Gegenbauer expansion of scalar mesons, we take into account the first two odd moments $B_{1}$ and $B_{3}$ in the twist 2 LCDAs [59] and the asymptotic terms in the twist 3 LCDAs due to the large theoretical uncertainty of $a_{m}$ and $b_{m}$ [60-62]. They are

\footnotetext{
${ }^{5}$ This result agrees with the previous LCSRs extractions from spacelike pion electromagnetic form factor [46], $B \rightarrow \pi$ form factor [47-49], and also the QCD sum rule prediction [50], but much larger than the recent lattice QCD evaluation $\left(a_{2}^{\pi}(1 \mathrm{GeV})=0.130\right)$ with the new developed momentum smearing technique [51].
}

$$
\begin{aligned}
& B_{1}^{a_{0}}=-0.93 \pm 0.10, \quad B_{3}^{a_{0}}=0.14 \pm 0.08, \\
& B_{1}^{a_{0}^{\prime}}=0.89 \pm 0.20, \quad B_{3}^{a_{0}^{\prime}}=-1.38 \pm 0.18
\end{aligned}
$$

in the first scenario, and

$$
\begin{aligned}
& B_{1}^{a_{0}^{\prime}}=-0.58 \pm 0.12, \quad B_{3}^{a_{0}^{\prime}}=-0.49 \pm 0.15, \\
& B_{1}^{a_{0}^{\prime \prime}}=0.73 \pm 0.45, \quad B_{3}^{a_{0}^{\prime \prime}}=0.17 \pm 0.20
\end{aligned}
$$

in the second scenario, where the default scale at $1 \mathrm{GeV}$ is indicated. 
TABLE I. Inputs of the single mesons (in units of $\mathrm{GeV}$ ) and the Wolfenstein parameters [42].

\begin{tabular}{|c|c|c|c|c|}
\hline$m_{B^{0}}=5.280$ & $m_{B^{ \pm}}=5.279$ & $m_{B_{s}^{0}}=5.367$ & $f_{B}=0.190$ & $f_{B_{s}}=0.230$ \\
\hline$m_{\pi^{ \pm}}=0.140$ & $m_{\pi^{0}}=0.135$ & $m_{K^{ \pm}}=0.494$ & $m_{K^{0}}=0.498$ & $m_{\eta}=0.548$ \\
\hline$f_{\pi^{ \pm}}=0.130$ & $f_{\pi^{0}}=0.156$ & $m_{a_{0}}=0.980$ & $m_{a_{0}^{\prime}}=1.474$ & $m_{a_{0}^{\prime \prime}}=1.931$ \\
\hline$\Gamma_{a_{0}}=0.075 \pm 0.025$ & & $\Gamma_{a_{0}^{\prime}}=0.265 \pm 0.013$ & \multicolumn{2}{|c|}{$\Gamma_{a_{0}^{\prime \prime}}=0.271 \pm 0.036[29]$} \\
\hline$\lambda=0.22650 \pm 0.00048$ & & $A=0.790_{-0.012}^{+0.017}$ & $\bar{\rho}=0.141_{-0.017}^{+0.016}$ & $\bar{\eta}=0.357 \pm 0.011$ \\
\hline
\end{tabular}

TABLE II. The PQCD predictions of branching fractions (in unit of $10^{-6}$ ) and $C P$ violations of $B \rightarrow a_{0}[\rightarrow K \bar{K} / \pi \eta] h$ decays in the first scenario of multiparticle configurations of $a_{0}$.

\begin{tabular}{|c|c|c|c|c|c|}
\hline Decay modes & Quasi-two-body & Narrow approximation & Two-body & Data [42] & $\mathrm{CPV}$ \\
\hline$B^{+} \rightarrow a_{0}^{+}\left[\rightarrow K^{+} \bar{K}^{0}\right] \pi^{0}$ & $0.08_{-0.03-0.00}^{+0.03+0.00}$ & & $0.41_{-0.23}^{+0.00}[63]$ & & $38.2_{-1.4-7.7}^{+3.5+3.5}$ \\
\hline$\rightarrow a_{0}^{+}\left[\rightarrow \pi^{+} \eta\right] \pi^{0}$ & $0.37_{-0.08-0.04}^{+0.03-0.04}$ & $0.52_{-0.11-0.05}^{+0.20+0.06}$ & $0.70_{-0.23}^{+0.32}[41]$ & $<1.4$ & $56.3_{-3.1-7.3}^{+1.4+2.8}$ \\
\hline$B^{+} \rightarrow a_{0}^{0}\left[\rightarrow K^{-} K^{+}\right] \pi^{+}$ & $0.33_{-0.08-0.04}^{+0.00+0.04}$ & & $2.8_{-1.3}^{+0.0}[63]$ & & $24.1_{-2.4-6.6}^{+2.6+6.5}$ \\
\hline$\rightarrow a_{0}^{0}\left[\rightarrow \pi^{0} \eta\right] \pi^{+}$ & $2.41_{-0.62-0.30}^{+0.01+0.37}$ & $3.44_{-0.88-0.42}^{+1.29+0.54}$ & $4.9_{-1.3}^{+1.4}[41]$ & $<5.8$ & $26.5_{-2.7-6.1}^{+0.1+5.4}$ \\
\hline$B^{+} \rightarrow a_{0}^{+}\left[\rightarrow K^{+} \bar{K}^{0}\right] K^{0}$ & $0.26_{-0.01-0.10}^{+0.03+0.16}$ & & $6.9_{-2.1}^{+2.4}[14]$ & & $\begin{array}{l}6.1_{-4.9-6.2}^{+5.5+5.4} \\
\end{array}$ \\
\hline$\rightarrow a_{0}^{+}\left[\rightarrow \pi^{+} \eta\right] K^{0}$ & $0.94_{-0.02-0.51}^{+0.04+0.85}$ & $1.35_{-0.03-0.72}^{+0.06+1.21}$ & $0.08_{-0.11}^{+2.20}[41]$ & $<3.9$ & $3.72_{-3.3-3.0}^{+2.4+5.1}$ \\
\hline$B^{+} \rightarrow a_{0}^{0}\left[\rightarrow K^{-} K^{+}\right] K^{+}$ & $0.11_{-0.0-0.04}^{+0.0+0.06}$ & & $3.5_{-1.2}^{+1.1}[14]$ & & $-26.4_{-4.2-6.7}^{+4.8+4.9}$ \\
\hline$\rightarrow a_{0}^{0}\left[\rightarrow \pi^{0} \eta\right] K^{+}$ & $1.06_{-0.04-0.42}^{-0.02+0.04}$ & $1.51_{-0.06-0.61}^{+0.03+0.85}$ & $0.34_{-0.16}^{+1.12}[41]$ & $<2.5$ & $-21.3_{-4.6-9.5}^{+4.0+7.4}$ \\
\hline$B^{0} \rightarrow a_{0}^{+}\left[\rightarrow K^{+} \bar{K}^{0}\right] \pi^{-}$ & $0.17_{-0.04-0.01}^{+0.06+0.01}$ & & $0.51_{-0.12}^{+0.12}[63]$ & & $70.5_{-3.1-7.4}^{+0.5+6.9}$ \\
\hline$\rightarrow a_{0}^{+}\left[\rightarrow \pi^{+} \eta\right] \pi^{-}$ & $0.67_{-0.15-0.07}^{+0.04+0.06}$ & $0.95_{-0.22-0.10}^{+0.34+0.08}$ & $0.58_{-0.25}^{+0.65}[41]$ & & $68.3_{-6.2-7.2}^{+3.4+6.4}$ \\
\hline$B^{0} \rightarrow a_{0}^{0}\left[\rightarrow K^{-} K^{+}\right] \pi^{0}$ & $0.04_{-0.01-0.00}^{+0.02+0.01}$ & & $0.51_{-0.11}^{+0.12}[63]$ & & $79.4_{-6.6-9.6}^{+0.4+7.9}$ \\
\hline$\rightarrow a_{0}^{0}\left[\rightarrow \pi^{0} \eta\right] \pi^{0}$ & $0.33_{-0.05-0.06}^{+0.09+0.05}$ & $0.47_{-0.07-0.08}^{+0.12+0.07}$ & $1.0_{-0.3}^{+0.5}[41]$ & & $84.1_{-56-5.9}^{+7.2+1.9}$ \\
\hline$B^{0} \rightarrow a_{0}^{-}\left[\rightarrow K^{-} K^{0}\right] \pi^{+}$ & $3.48_{-0.92-0.29}^{+1.33+0.34}$ & & $0.86_{-0.17}^{+0.17}[63]$ & & $17.8_{-2.2-3.5}^{+2.3+3.1}$ \\
\hline$\rightarrow a_{0}^{-}\left[\rightarrow \pi^{-} \eta\right] \pi^{+}$ & $14.8_{-3.9-1.4}^{+5.6+1.7}$ & $21.1_{-5.6-2.1}^{+7.9+2.3}$ & $5.3_{-1.4}^{+1.7}[41]$ & & $20.6_{-2.7-3.6}^{+2.6+2.7}$ \\
\hline$B^{0} \rightarrow a_{0}^{0}\left[\rightarrow K^{-} K^{+}\right] K^{0}$ & $0.11_{-0.01-0.02}^{+0.93+0.4}$ & & $4.7_{-1.4}^{+1.4}[14]$ & & $-27.5_{-1.7-2.1}^{+6.9+5.6}$ \\
\hline$\rightarrow a_{0}^{0}\left[\rightarrow \pi^{0} \eta\right] K^{0}$ & $1.36_{-0.23-0.51}^{+0.21+0.43}$ & $1.95_{-0.32-0.72}^{+0.30+0.61}$ & $0.05_{-0.05}^{+0.91}[41]$ & $<7.8$ & $-43.2_{-7.8-8.8}^{+1.7+5.7}$ \\
\hline$B^{0} \rightarrow a_{0}^{-}\left[\rightarrow K^{-} K^{0}\right] K^{+}$ & $0.99_{-0.09-0.33}^{+0.14+0.38}$ & & $9.7_{-2.8}^{+3.3}[14]$ & & $-69.7_{-4.1-2.6}^{+1.0+1.7}$ \\
\hline$\rightarrow a_{0}^{-}\left[\rightarrow \pi^{-} \eta\right] K^{+}$ & $4.51_{-0.61-1.60}^{+0.60+1.72}$ & $6.44_{-0.87-2.33}^{+0.85+2.53}$ & $0.34_{-0.14}^{+2.35}[41]$ & $<1.9$ & $-83.2_{-9.6-9.9}^{+2.5+3.4}$ \\
\hline
\end{tabular}

Our numerical evaluations are carried out in two scenarios. In the first scenario, we treat $a_{0}$ as the lowestlying $q \bar{q}$ state and $a_{0}^{\prime}$ as its first excited state, and study the contributions from $a_{0}$ and $a_{0}^{\prime}$ in the $B \rightarrow$ $a_{0}^{(\prime)}[\rightarrow K \bar{K} / \pi \eta] h$ decays. The second scenario indicates that $a_{0}^{\prime}$ is the lowest-lying $q \bar{q}$ state and $a_{0}^{\prime \prime}$ is the first excited state, with this ansatz we study their contributions in the $B \rightarrow a_{0}^{\prime \prime \prime \prime}[\rightarrow K \bar{K} / \pi \eta] h$ decays.

In Table II and Table III, we present the PQCD predictions of $B \rightarrow a_{0}[\rightarrow K \bar{K} / \pi \eta] h$ and $B \rightarrow a_{0}^{\prime}[\rightarrow K \bar{K} / \pi \eta] h$ decays in the first scenario of multiparticle configurations of $a_{0}$, respectively. Besides the result of quasi-two-body decays, say the branching fractions (in the 2 nd column) and the $C P$ violations (in the last column), we list the branching fractions of two-body $B \rightarrow a_{0}^{\prime} h$ decays ${ }^{6}$ obtained in the narrow width approximation (in the 3rd column), for the

\footnotetext{
${ }^{6}$ The narrow width approximation is not applicable to the modes involving $a_{0} h \rightarrow K K$ due to the threshold effect, so in Table II we do not list the result of two-body $B \rightarrow a_{0} h$ decay
}

sake of comparison, the direct two-body calculations based on PQCD [14] and QCDF approach [41], and also the available data are listed too (in the 4th and 5th columns). The theoretical uncertainties come from the inputs of LCDAs, mainly from the inverse moment $\omega_{B}$ which we put as the first error source, the uncertainties from Gegenbauer moments $B_{1}^{a_{0}}, B_{3}^{a_{0}}$ of dimeson systems are added together as the second error, we do not consider the uncertainty from other parameters, like $f_{a_{0}}, \bar{f}_{a_{0}}$ since their influences are small. We comment in orders,

(a) The branching fractions of quasi-two-body channels with strong decays $a_{0} \rightarrow \pi \eta$ is about 5 times larger than that with the strong decay $a_{0} \rightarrow K \bar{K}$, which is understood by the suppressed phase space for $K \bar{K}$ state.

(b) Under the narrow width approximation of the quasitwo-body decays, we extract the branching fractions of relevant two body decays $B \rightarrow a_{0}^{(\prime)} h$. The result obtained from the $a_{0}^{\prime} \rightarrow K \bar{K}$ and $a_{0}^{\prime} \rightarrow \pi \eta$ modes are consist with each other with in the uncertainties, more important is that this result have a large discrepancy with the direct two-body calculation from 
TABLE III. The same as Table II, but for the $B \rightarrow a_{0}^{\prime}[\rightarrow K \bar{K} / \pi \eta] h$ decays.

\begin{tabular}{|c|c|c|c|c|}
\hline Decay modes & Quasi-two-body & Narrow approximation & Two-body [41] & $\mathrm{CPV}$ \\
\hline$B^{+} \rightarrow a_{0}^{\prime+}\left[\rightarrow K^{+} \bar{K}^{0}\right] \pi^{0}$ & $0.08_{-0.02-0.01}^{+0.01+0.00}$ & $0.94_{-0.20-0.19}^{+0.12+0.03}$ & & $-4.6_{-9.2-9.1}^{+1.9+4.5}$ \\
\hline$\rightarrow a_{0}^{\prime+}\left[\rightarrow \pi^{+} \eta\right] \pi^{0}$ & $0.09_{-0.02-0.01}^{+0.02+0.01}$ & $0.95_{-0.21-0.16}^{+0.20 .18}$ & $0.4_{-0.3}^{+0.3}$ & $-13.2_{-12.7-8.3}^{+9.1+7.8}$ \\
\hline$B^{+} \rightarrow a_{0}^{\prime 0}\left[\rightarrow K^{-} K^{+}\right] \pi^{+}$ & $0.12_{-0.03-0.02}^{+0.04+0.03}$ & $2.81_{-0.61-0.54}^{+1.09+0.73}$ & & $26.6_{-19.9-17.0}^{+23.7+13.3}$ \\
\hline$\rightarrow a_{0}^{\prime 0}\left[\rightarrow \pi^{0} \eta\right] \pi^{+}$ & $0.28_{-0.07-0.04}^{+0.010+0.07}$ & $3.02_{-0.74-0.52}^{+1.09+0.78}$ & $2.7_{-0.7}^{+0.7}$ & $28.2_{-16.7-18.4}^{+14.6+13.0}$ \\
\hline$B^{+} \rightarrow a_{0}^{\prime+}\left[\rightarrow K^{+} \bar{K}^{0}\right] K^{0}$ & $1.28_{-0.05-0.40}^{+0.03+0.45}$ & $15.6_{-0.6-2.2}^{+0.4+2.7}$ & & $4.8_{-0.5-3.1}^{+1.5+2.9}$ \\
\hline$\rightarrow a_{0}^{\prime+}\left[\rightarrow \pi^{+} \eta\right] K^{0}$ & $1.50_{-0.06-0.48}^{+0.004+0.53}$ & $16.1_{-05-5.0}^{+0.0-5+.7}$ & $2.7_{-3.2}^{+10.1}$ & $4.8_{-0.3-0.6}^{+1.4+1.9}$ \\
\hline$B^{+} \rightarrow a_{0}^{\prime 0}\left[\rightarrow K^{-} K^{+}\right] K^{+}$ & $0.44_{-0.01-0.14}^{+0.01+0.16}$ & $10.8_{-0.4-2.6}^{+0.3+2.9}$ & & $1.0_{-0.8-3.2}^{+0.1+5.9}$ \\
\hline$\rightarrow a_{0}^{\prime 0}\left[\rightarrow \pi^{0} \eta\right] K^{+}$ & $1.02_{-0.03-0.34}^{+0.03+0.34}$ & $11.1_{-0.4-3.6}^{+0.2+4.0}$ & $0.7_{-0.6}^{+3.2}$ & $0.8_{-0.6-3.0}^{+0.5+6.2}$ \\
\hline$B^{0} \rightarrow a_{0}^{\prime+}\left[\rightarrow K^{+} \bar{K}^{0}\right] \pi^{-}$ & $0.04_{-0.01-0.01}^{+0.01+0.01}$ & $0.49_{-0.08-0.14}^{+0.14+0.17}$ & & $-24.0_{-13.0-18.2}^{+12.0+19.3}$ \\
\hline$\rightarrow a_{0}^{\prime+}\left[\rightarrow \pi^{+} \eta\right] \pi^{-}$ & $0.03_{-0.00-0.01}^{+0.01+0.01}$ & $0.36_{-0.03-0.12}^{+0.10+17}$ & $0.02_{-0.01}^{+0.75}$ & $-20.7_{-10.7-23.0}^{+15.6+25.4}$ \\
\hline$B^{0} \rightarrow a_{0}^{\prime 0}\left[\rightarrow K^{-} K^{+}\right] \pi^{0}$ & $0.03_{-0.01-0.01}^{+0.01+0.01}$ & $0.67_{-0.12-0.15}^{+0.016+0.21}$ & & $-22.0_{-13.1-19.6}^{+19.1+18.0}$ \\
\hline$\rightarrow a_{0}^{\prime 0}\left[\rightarrow \pi^{0} \eta\right] \pi^{0}$ & $0.07_{-0.01-0.02}^{-0.01+0.01}$ & $0.70_{-0.08-0.23}^{+0.16+0.18}$ & $1.3_{-1.1}^{+2.1}$ & $-31.9_{-85-19.2}^{+13.4+19.1}$ \\
\hline$B^{0} \rightarrow a_{0}^{\prime-}\left[\rightarrow K^{-} K^{0}\right] \pi^{+}$ & $1.08_{-0.24-0.17}^{+0.018}$ & $13.2_{-2.9-2.2}^{+4.2+2.2}$ & & $24.8_{-0.7-5.3}^{+1.2+4.4}$ \\
\hline$\rightarrow a_{0}^{\prime-}\left[\rightarrow \pi^{-} \eta\right] \pi^{+}$ & $1.24_{-0.27-0.21}^{-0.24+0.211}$ & $13.3_{-2.9-2.2}^{+4.3+2.2}$ & $11.2_{-5.7}^{+5.2}$ & $25.6_{-0.8-5.9}^{+2.9+4.3}$ \\
\hline$B^{0} \rightarrow a_{0}^{\prime 0}\left[\rightarrow K^{-} K^{+}\right] K^{0}$ & $0.25_{-0.02-0.09}^{+0.02+0.12}$ & $6.06_{-0.56-2.28}^{+0.50+3.01}$ & & $-0.3_{-4.9-0.4}^{+2.9-3+.9}$ \\
\hline$\rightarrow a_{0}^{0}\left[\rightarrow \pi^{0} \eta\right] K^{0}$ & $0.58_{-0.05-0.22}^{+0.02+0.09}$ & $6.27_{-0.52-2.39}^{+0.52+3.14}$ & $0.9_{-1.1}^{+3.8}$ & $-0.6_{-65-0.8}^{+3.4+2.4}$ \\
\hline$B^{0} \rightarrow a_{0}^{\prime-}\left[\rightarrow K^{-} K^{0}\right] K^{+}$ & $2.62_{-0.29-0.62}^{+0.031+0.72}$ & $32.0_{-3.4-4.7}^{+3.8+6.7}$ & & $-18.9_{-2.3-3.8}^{-0.7+1.8}$ \\
\hline$\rightarrow a_{0}^{\prime-}\left[\rightarrow \pi^{-} \eta\right] K^{+}$ & $3.04_{-0.33-0.73}^{+0.0 .35}$ & $\begin{array}{l}32.7_{-3.6-6.6}^{+3.4-7.7} \\
0\end{array}$ & $1.9_{-1.8}^{+8.1}$ & $-19.5_{-2.2-4.0}^{+2.0+0.8}$ \\
\hline
\end{tabular}

PQCD [14] and QCDF [41], revealing the important role of width effects of $a_{0}$ and $a_{0}^{\prime}$.

(c) In the $B \rightarrow a_{0}^{\prime} h$ and the following $B \rightarrow a_{0}^{\prime \prime} h$ decays, only the partial width expression is used due to the lack of direct measurements, that is why the branching fractions of these decays extracted from $K \bar{K}$ and $\pi \eta$ modes are very close to each other.

(d) The PQCD predictions of branching fractions of the six $B \rightarrow a_{0}^{(+, 0)}[\rightarrow \pi \eta] h$ quasi-two-body decays do not excess the experimental upper limit, the $\mathrm{p}$ redictions of two channels $B^{0} \rightarrow a_{0}^{ \pm}\left[\rightarrow \pi^{ \pm} \eta\right] \pi^{\mp}$ excess the experimental upper limit $3.1 \times 10^{-6}$ [42] at first glance, but the large uncertainties would be larger if we consider the uncertainty of $\omega_{B}=440 \pm 110 \mathrm{MeV}$. Within acceptable limits, the $q \bar{q}$ configuration of $a_{0}$ is not ruled out in $B$ decays. It is shown that the decaying channel $B^{0} \rightarrow a_{0}^{-}\left[\rightarrow \pi^{-} \eta\right] \pi^{+}$has the largest branching fraction, and we suggest the measurement to examine the $q \bar{q}$ configuration.

We list in Table IV and Table $\mathrm{V}$ with the PQCD predictions of $B \rightarrow a_{0}^{\prime}[\rightarrow K \bar{K} / \pi \eta] h$ and $B \rightarrow a_{0}^{\prime \prime}[\rightarrow K \bar{K} / \pi \eta] h$ decays in the second scenario of multiparticle configurations of $a_{0}$, respectively. For the latter one, we also present the uncertainty (as the third error) in the quasi-two-body decays from the partial decay width $\Gamma_{a_{0}^{\prime \prime} \rightarrow K \bar{K} / \pi \eta}$ as demonstrated in Eq. (22), this parameter would not bring additional uncertainty to the two-body decays under narrow approximation. A similar result is obtained with showing that the decaying channels $B^{0} \rightarrow a_{0}^{\prime-}\left[\rightarrow K^{-} K^{0} / \pi^{-} \eta\right] h$ have the largest branching fractions both for the quasi-two-body and the extracted two-body decays. We would like to mark that our predictions of the $a_{0}^{\prime}$ contributions are comparable in most of the $B \rightarrow K \bar{K} h, \pi \eta h$ decays no matter what the scenarios of $a_{0}$ configurations are taken, while for the channels $B^{0} \rightarrow a_{0}^{\prime+}\left[\rightarrow K^{+} \bar{K}^{0}, \pi^{+} \eta\right] \pi^{-}$and $B^{0} \rightarrow a_{0}^{\prime-}\left[\rightarrow K^{-} K^{0}, \pi^{-} \eta\right] \pi^{+}$, the predictions of branching fractions in the second scenario are about three times larger in magnitude than that predicted in the first scenario, ${ }^{7}$ which provide another opportunity to check which one is correct with the future measurement. In these tables we also list $C P$ violations which provide another observable to study the interactions between different operators and/or topological amplitudes, especially the different sources of strong phases.

The width effect of intermediate isovector scalar mesons is exhibited explicitly by the $K \bar{K} / \pi \eta$ invariant mass spectral. In the first scenario of multiparticle configurations of $a_{0}$, we plot in Fig. 2 for the differential branching fractions of the typical $B \rightarrow a_{0}^{(\prime)}[\rightarrow K \bar{K} / \pi \eta] h$ decaying channels on the invariant masses, in which the top panel shows the result of channels $B^{+} \rightarrow a_{0}^{0}\left[\rightarrow K^{+} K^{-} / \pi^{0} \eta\right] \pi^{+}$ (left) and $B^{+} \rightarrow a_{0}^{+}\left[\rightarrow K^{+} \bar{K}^{0} / \pi^{+} \eta\right] K^{0}$ (right) with varying the invariant mass from thresholds to $2.0 \mathrm{GeV}$, the medium panel is the result of $B^{+} \rightarrow a_{0}^{\prime 0}\left[\rightarrow K^{+} K^{-} / \pi^{0} \eta\right] \pi^{+}$(left) and $B^{+} \rightarrow a_{0}^{++}\left[\rightarrow K^{+} \bar{K}^{0} / \pi^{+} \eta\right] K^{0}$ (right) decays with varying

\footnotetext{
${ }^{7}$ The PQCD predictions in the second scenario for these channels consist of the result from the factorization approach under $S U(3)$ symmetry [64], and the predictions in both scenarios are under the experiment's upper limit.
} 
TABLE IV. The PQCD predictions of branching fractions (in unit of $10^{-6}$ ) and $C P$ violations of $B \rightarrow a_{0}^{\prime}[\rightarrow K \bar{K} / \pi \eta] h$ decays in the second scenario of multiparticle configurations of $a_{0}$.

\begin{tabular}{|c|c|c|c|c|}
\hline Decay modes & Quasi-two-body & Narrow approximation & Two-body [41] & $\mathrm{CPV}$ \\
\hline$B^{+} \rightarrow a_{0}^{\prime+}\left[\rightarrow K^{+} \bar{K}^{0}\right] \pi^{0}$ & $0.10_{-0.03-0.01}^{+0.04+0.00}$ & $1.24_{-0.34-0.09}^{+0.52+0.03}$ & & $-19.2_{-4.3-8.1}^{+4.9+5.1}$ \\
\hline$\rightarrow a_{0}^{\prime+}\left[\rightarrow \pi^{+} \eta\right] \pi^{0}$ & $0.12_{-0.03-0.01}^{+0.05+0.01}$ & $1.24_{-0.31-0.10}^{+0.53+0.08}$ & $2.1_{-0.8}^{+1.1}$ & $-15.2_{-3.1-6.8}^{+2.4+5.3}$ \\
\hline$B^{+} \rightarrow a_{0}^{\prime 0}\left[\rightarrow K^{-} K^{+}\right] \pi^{+}$ & $0.25_{-0.07-0.04}^{+0.011+0.04}$ & $6.07_{-1.77-0.98}^{+2.80+1.06}$ & & $-0.1_{-1.5-2.0}^{+1.6+3.6}$ \\
\hline$\rightarrow a_{0}^{\prime 0}\left[\rightarrow \pi^{0} \eta\right] \pi^{+}$ & $0.56_{-0.16-0.09}^{+0.07+0.09}$ & $6.01_{-1.72-0.99}^{+2.91+1.08}$ & $5.1_{-1.7}^{+1.8}$ & $1.0_{-2.6-3.7}^{+0.8+3.4}$ \\
\hline$B^{+} \rightarrow a_{0}^{\prime+}\left[\rightarrow K^{+} \bar{K}^{0}\right] K^{0}$ & $1.29_{-0.02-0.52}^{+0.03+0.68}$ & $15.8_{-0.2-3.9}^{+0.4+4.6}$ & & $0.5_{-0.1-0.6}^{+0.1+0.6}$ \\
\hline$\rightarrow a_{0}^{\prime+}\left[\rightarrow \pi^{+} \eta\right] K^{0}$ & $1.51_{-0.03-0.62}^{+0.04+0.79}$ & $16.3_{-0.3-6.5}^{+0.5+8.9}$ & $4.2_{-4.8}^{+18.8}$ & $0.3_{-0.3-0.9}^{+0.2+0.0}$ \\
\hline$B^{+} \rightarrow a_{0}^{\prime 0}\left[\rightarrow K^{-} K^{+}\right] K^{+}$ & $0.50_{-0.01-0.23}^{+0.00+0.23}$ & $12.3_{-0.3}^{+0.1+3.9}$ & & $-22.7_{-3}^{+2.4+1.3}$ \\
\hline$\rightarrow a_{0}^{\prime 0}\left[\rightarrow \pi^{0} \eta\right] K^{+}$ & $1.13_{-0.02-0.43}^{+0.00+0.56}$ & $12.2_{-0.1-4.7}^{+0.1+5.9}$ & $2.2_{-2.2}^{+8.1}$ & $-23.6_{-2.0-1.8}^{+3.0+3.8}$ \\
\hline$B^{0} \rightarrow a_{0}^{\prime+}\left[\rightarrow K^{+} \bar{K}^{0}\right] \pi^{-}$ & $0.13_{-0.03-0.01}^{+0.05+0.01}$ & $1.56_{-0.37-0.13}^{+0.57+0.18}$ & & $24.8_{-0.6-6.8}^{+0.5+6.5}$ \\
\hline$\rightarrow a_{0}^{\prime+}\left[\rightarrow \pi^{+} \eta\right] \pi^{-}$ & $0.14_{-0.03-0.01}^{+0.05+0.01}$ & $1.51_{-0.37-0.12}^{+0.57+0.16}$ & $0.74_{-0.6}^{+2.9}$ & $28.5_{-0.5-4.6}^{+0.4+6.0}$ \\
\hline$B^{0} \rightarrow a_{0}^{\prime 0}\left[\rightarrow K^{-} K^{+}\right] \pi^{0}$ & $0.05_{-0.01-0.01}^{+0.03-0.01}$ & $1.07_{-0.10-0.32}^{+0.316+0.34}$ & & $26.1_{-6.7-8.6}^{+0.5-4.0}$ \\
\hline$\rightarrow a_{0}^{\prime 0}\left[\rightarrow \pi^{0} \eta\right] \pi^{0}$ & $0.10_{-0.01-0.03}^{+0.01+0.01}$ & $1.10_{-0.11-0.34}^{+0.15+0.34}$ & $3.3_{-1.7}^{+3.1}$ & $24.3_{-6.4-12.3}^{+6.5+17.2}$ \\
\hline$B^{0} \rightarrow a_{0}^{\prime-}\left[\rightarrow K^{-} K^{0}\right] \pi^{+}$ & $3.61_{-0.92-0.36}^{+1.01-0.03}$ & $44.0_{-112-4.4}^{-16.11-4.34}$ & & $25.8_{-3.0-36}^{+3.3+4.0}$ \\
\hline$\rightarrow a_{0}^{\prime-}\left[\rightarrow \pi^{-} \eta\right] \pi^{+}$ & $4.15_{-1.05-0.42}^{+1.52-0.30}$ & $44.6_{-11.4-4.5}^{+116.4+4.9}$ & $2.5_{-1.0}^{+3.8}$ & $26.1_{-2.9-3.6}^{+3.3+3.0}$ \\
\hline$B^{0} \rightarrow a_{0}^{\prime 0}\left[\rightarrow K^{-} K^{+}\right] K^{0}$ & $0.33_{-0.00-0.13}^{+0.01+0.17}$ & $8.10_{-0.02-3.01}^{+0.16+4.05}$ & & $-6.3_{-2.5-3.1}^{+0.1+0.8}$ \\
\hline$\rightarrow a_{0}^{\prime 0}\left[\rightarrow \pi^{0} \eta\right] K^{0}$ & $0.78_{-0.00-0.29}^{+0.001+0.40}$ & $8.34_{-0.02-3.96}^{+0.17+4.01}$ & $1.9_{-2.2}^{+7.8}$ & $-7.5_{-2.3-2.4}^{+0.7+1.1}$ \\
\hline$B^{0} \rightarrow a_{0}^{\prime-}\left[\rightarrow K^{-} K^{0}\right] K^{+}$ & $2.93_{-0.35-0.89}^{+0.00-0.29}$ & $\begin{array}{c}35.7_{-4.2-9.7}^{+6.02-3.96} \\
\end{array}$ & & $-46.7_{-0.3-3.6}^{+1.6+4.1}$ \\
\hline$\rightarrow a_{0}^{\prime-}\left[\rightarrow \pi^{-} \eta\right] K^{+}$ & $3.39_{-0.39-1.02}^{+0.52+1.02}$ & $36.5_{-4.2-10.0}^{+5.6+13.0}$ & $3.5_{-3.9}^{+17.5}$ & $-46.0_{-1.5-4.1}^{+3.3+4.0}$ \\
\hline
\end{tabular}

TABLE V. The same as Table IV, but for the $B \rightarrow a_{0}^{\prime \prime}[\rightarrow K \bar{K} / \pi \eta] h$ decays.

\begin{tabular}{|c|c|c|c|}
\hline Decay modes & Quasi-two-body & Narrow approximation & $\mathrm{CPV}$ \\
\hline$B^{+} \rightarrow a_{0}^{\prime \prime+}\left[\rightarrow K^{+} \bar{K}^{0}\right] \pi^{0}$ & $0.38_{-0.10-0.02}^{+0.17+0.02} \pm 0.22$ & $1.14_{-0.30-0.04}^{+0.50+0.06}$ & $17.6_{-4.1-0.8}^{+1.9+0.8}$ \\
\hline$\rightarrow a_{0}^{\prime \prime+}\left[\rightarrow \pi^{+} \eta\right] \pi^{0}$ & $0.39_{-0.10-0.01}^{+0.16+0.01} \pm 0.07$ & $1.16_{-0.31-0.04}^{+0.48+0.03}$ & $13.3_{-0.1-0.7}^{+3.1+0.9}$ \\
\hline$B^{+} \rightarrow a_{0}^{\prime \prime 0}\left[\rightarrow K^{-} K^{+}\right] \pi^{+}$ & $3.04_{-0.81-0.48}^{+1.22+0.59} \pm 1.75$ & $18.1_{-4.8-2.8}^{+7.3+3.5}$ & $-6.7_{-1.3-2.2}^{+1.1+2.1}$ \\
\hline$\rightarrow a_{0}^{\prime \prime 0}\left[\rightarrow \pi^{0} \eta\right] \pi^{+}$ & $6.31_{-0.71-1.07}^{+2.48+1.20} \pm 1.07$ & $18.8_{-5.1-3.3}^{+7.4+3.5}$ & $-7.0_{-0.9-1.7}^{+0.9+0.9}$ \\
\hline$B^{+} \rightarrow a_{0}^{\prime \prime+}\left[\rightarrow K^{+} \bar{K}^{0}\right] K^{0}$ & $2.60_{-0.08-2.01}^{+0.15+3.18} \pm 1.49$ & $7.73_{-0.25-6.01}^{+0.37+9.49}$ & $0.6_{-0.7-0.6}^{+0.0+1.4}$ \\
\hline$\rightarrow a_{0}^{\prime \prime+}\left[\rightarrow \pi^{+} \eta\right] K^{0}$ & $2.62_{-0.09-2.04}^{+0.15+3.21} \pm 0.44$ & $7.81_{-0.25-6.09}^{+0.44+9.59}$ & $0.5_{-0.8-0.3}^{+0.4+0.7}$ \\
\hline$B^{+} \rightarrow a_{0}^{\prime \prime 0}\left[\rightarrow K^{-} K^{+}\right] K^{+}$ & $0.58_{-0.00-0.52}^{+0.00+0.83} \pm 0.33$ & $3.46_{-0.01-3.13}^{+0.02+4.96}$ & $-27.9_{-4.2-6.8}^{+3.8+6.3}$ \\
\hline$\rightarrow a_{0}^{\prime \prime 0}\left[\rightarrow \pi^{0} \eta\right] K^{+}$ & $1.19_{-0.00-1.07}^{+0.01+1.66} \pm 0.20$ & $3.56_{-0.05-3.13}^{+0.01+4.95}$ & $-30.8_{-5.4-7.0}^{+4.4+7.3}$ \\
\hline$B^{0} \rightarrow a_{0}^{\prime \prime+}\left[\rightarrow K^{+} \bar{K}^{0}\right] \pi^{-}$ & $1.02_{-0.23-0.21}^{+0.35+0.27} \pm 0.59$ & $3.05_{-0.72-0.66}^{+1.03+0.80}$ & $-7.0_{-2.6-8.5}^{+2.5+8.4}$ \\
\hline$\rightarrow a_{0}^{\prime \prime+}\left[\rightarrow \pi^{+} \eta\right] \pi^{-}$ & $1.01_{-0.23-0.20}^{+0.35+0.27} \pm 0.17$ & $3.02_{-0.70-0.64}^{+1.04+0.83}$ & $-7.8_{-2.2-7.9}^{+3.2+8.8}$ \\
\hline$B^{0} \rightarrow a_{0}^{\prime \prime 0}\left[\rightarrow K^{-} K^{+}\right] \pi^{0}$ & $0.22_{-0.05-0.10}^{+0.06+0.11} \pm 0.13$ & $1.32_{-0.27-0.59}^{+0.33+0.69}$ & $-31.3_{-2.9-8.9}^{+1.2+5.7}$ \\
\hline$\rightarrow a_{0}^{\prime \prime 0}\left[\rightarrow \pi^{0} \eta\right] \pi^{0}$ & $0.44_{-0.08-0.19}^{+0.12+0.24} \pm 0.07$ & $1.30_{-0.25-0.56}^{+0.36+0.72}$ & $-32.6_{-1.8-8.1}^{+2.7+7.5}$ \\
\hline$B^{0} \rightarrow a_{0}^{\prime \prime-}\left[\rightarrow K^{-} K^{0}\right] \pi^{+}$ & $4.76_{-1.34-1.19}^{+2.03+1.45} \pm 2.73$ & $14.2_{-4.0-3.7}^{+8.0+4.2}$ & $-24.2_{-3.9-10.7}^{+3.6+11.5}$ \\
\hline$\rightarrow a_{0}^{\prime \prime-}\left[\rightarrow \pi^{-} \eta\right] \pi^{+}$ & $4.76_{-1.34-1.19}^{+2.04+1.46} \pm 0.81$ & $14.2_{-4.1-3.7}^{+8.0+4.2}$ & $-24.3_{-4.0-10.1}^{+3.5+9.7}$ \\
\hline$B^{0} \rightarrow a_{0}^{\prime \prime 0}\left[\rightarrow K^{-} K^{+}\right] K^{0}$ & $0.86_{-0.13-0.67}^{+0.22+0.73} \pm 0.50$ & $5.15_{-0.80-3.05}^{+1.33+4.40}$ & $-1.8_{-0.6-1.8}^{+1.4+0.1}$ \\
\hline$\rightarrow a_{0}^{\prime \prime 0}\left[\rightarrow \pi^{0} \eta\right] K^{0}$ & $1.74_{-0.26-1.04}^{+0.43+1.48} \pm 0.29$ & $5.20_{-0.79-3.12}^{+1.29+4.42}$ & $-2.1_{-0.2-1.1}^{+1.2+0.2}$ \\
\hline$B^{0} \rightarrow a_{0}^{\prime \prime-}\left[\rightarrow K^{-} K^{0}\right] K^{+}$ & $3.82_{-0.67-1.32}^{+1.01+2.11} \pm 2.19$ & $11.4_{-2.0-3.9}^{+2.9+6.2}$ & $24.9_{-0.4-4.0}^{+0.7+5.5}$ \\
\hline$\rightarrow a_{0}^{\prime \prime-}\left[\rightarrow \pi^{-} \eta\right] K^{+}$ & $3.80_{-0.64-1.31}^{+1.02+2.18} \pm 0.65$ & $11.3_{-1.8-3.8}^{+3.1+6.7}$ & $25.6_{-0.6-9.7}^{+1.2+8.5}$ \\
\hline
\end{tabular}

the invariant mass from thresholds to $3.0 \mathrm{GeV}$, the comparison of $a_{0}$ and $a_{0}^{\prime}$ contributions in $B^{+} \rightarrow\left[\pi^{0} \eta\right] \pi^{+}$ (left) and $B^{+} \rightarrow\left[\pi^{+} \eta\right] K^{0}$ (right) decays is depicted in the bottom panel. We take these typical charged channels because they carry almost all the characteristics of the relevant quasi-two-body decays: (a) the $a_{0}$ contribution from $K \bar{K}$ mode is much smaller than it from $\pi \eta$ modes as expected by the highly phase space suppression ${ }^{8}$; (b) the $a_{0}^{\prime}$

\footnotetext{
${ }^{8}$ We multiply the result of the $K \bar{K}$ mode by a factor of ten to show apparently for the curves.
} 

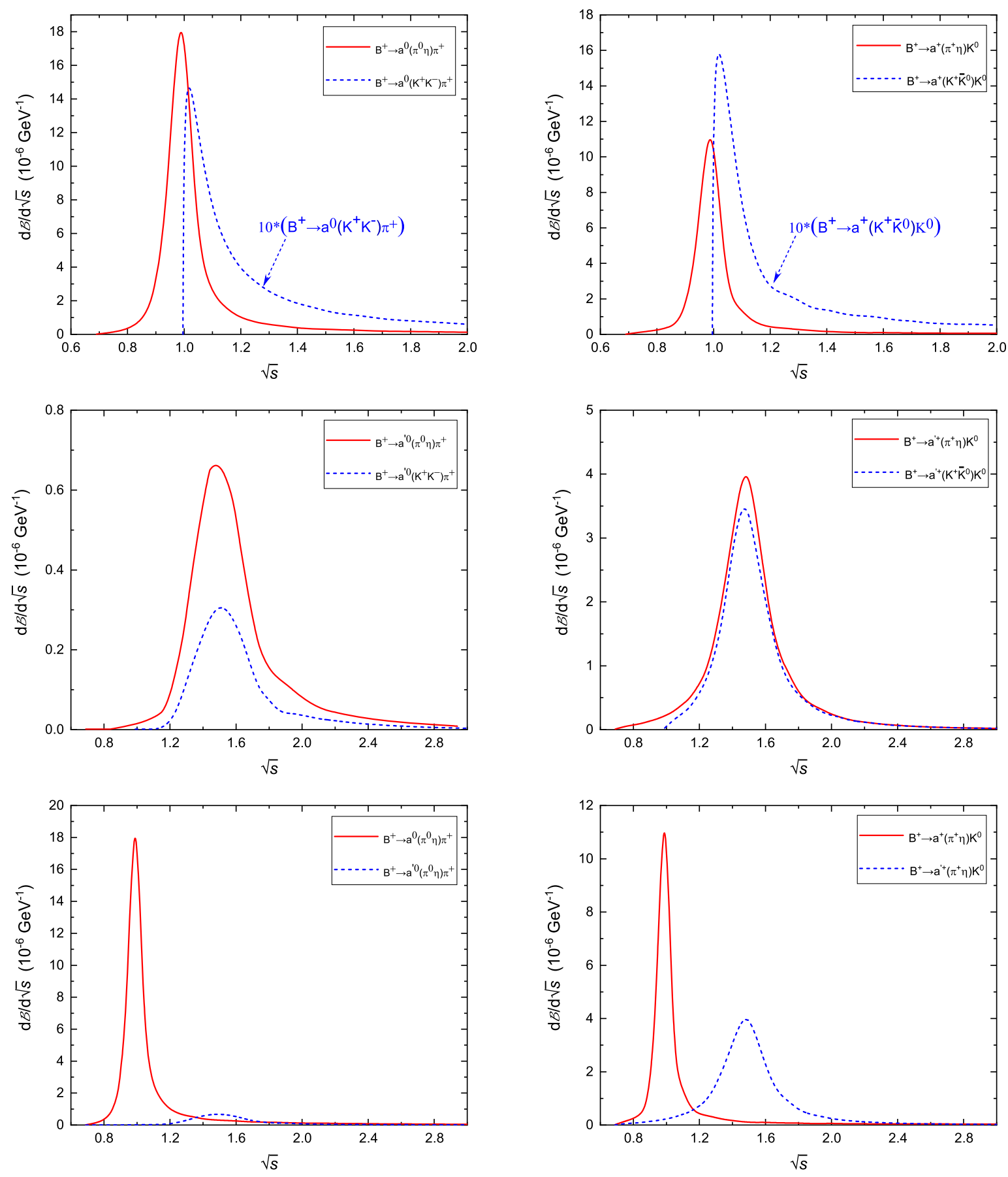

FIG. 2. Differential branching fractions of typical $B \rightarrow a_{0}^{(\prime)}[\rightarrow K \bar{K} / \pi \eta] h$ decays in the first scenario of multiparticle configurations of $a_{0}$ mesons.

contributions from these two modes are comparable, we comment that the lower curves in the left plot can be compensated by the channel $B^{+} \rightarrow a_{0}^{\prime 0}\left[\rightarrow K^{0} \bar{K}^{0}\right] \pi^{+}$which is not depicted here; (c) in contrast to the $a_{0}$ contributions, the $a_{0}^{\prime}$ contribution is negligible in the $\left[\pi^{0} \eta\right] \pi^{+}$channel and small in the $\left[\pi^{+} \eta\right] K^{0}$ channel, while its contributions in the $\left[K^{+} K^{-}\right] \pi^{+}$and $\left[K^{+} \bar{K}^{0}\right] K^{0}$ channels are (much) larger than the contributions from $a_{0}$, this is mainly decided by the different phase spaces. We can also see the difference between the three plots in the left panel for the channels with $h=\pi$ and the other three plots on the right panel for the channels with $h=K$, this is determined by the weak decay of relevant two-body decays $B^{+} \rightarrow a_{0}^{(\prime)} \pi$ and $B^{+} \rightarrow$ $a_{0}^{(\prime)} K$ whose invariant amplitudes are collected in the Appendix B. These points support the corresponding result in Tables II and III for the partial decay branching fractions obtained by integrating the differential branching fractions over the invariant masses. 

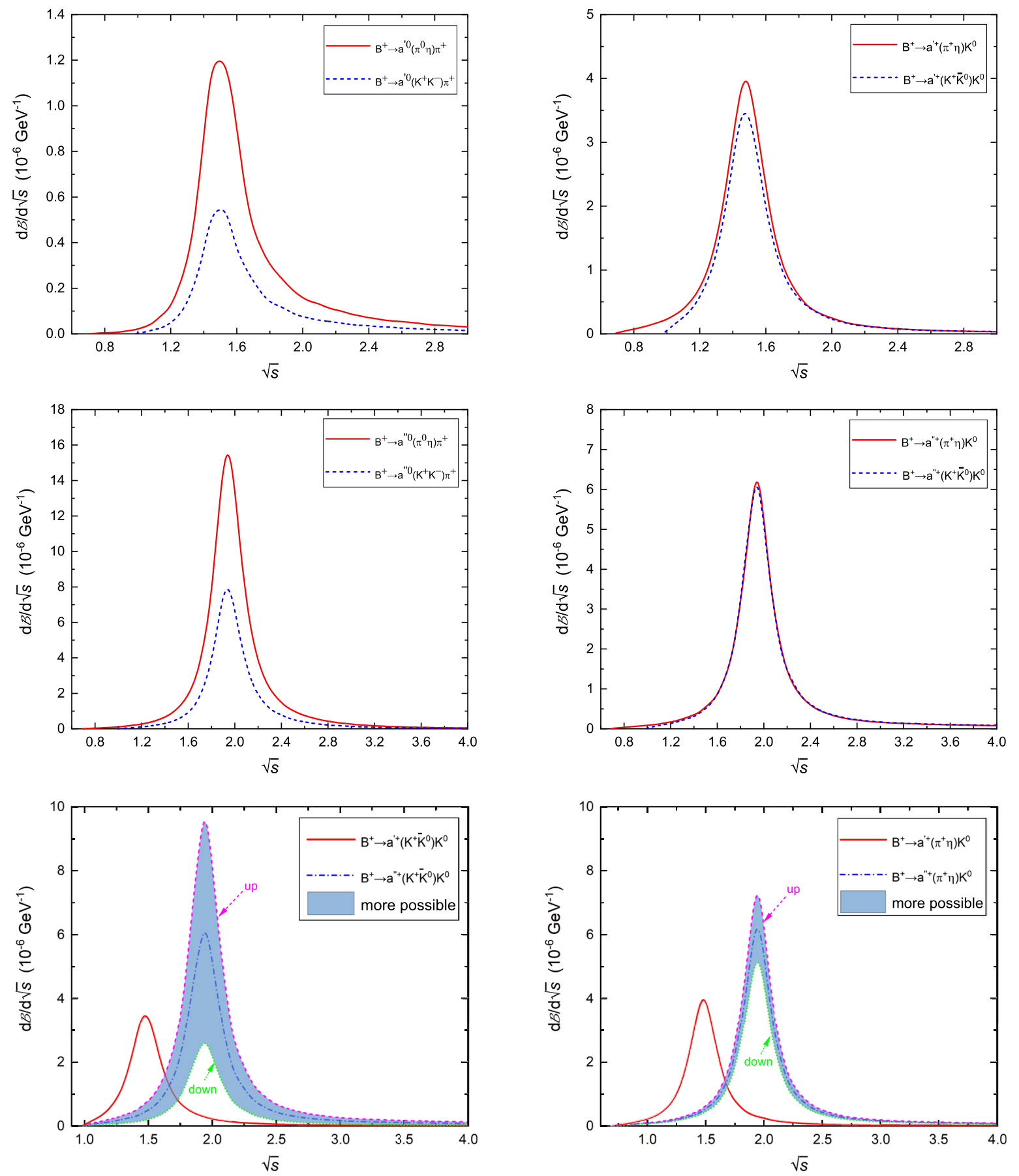

FIG. 3. Differential branching fractions of typical $B \rightarrow a_{0}^{\prime \prime \prime \prime}[\rightarrow K \bar{K} / \pi \eta] h$ decays in the second scenario of multiparticle configurations of $a_{0}$ mesons.

We similarly plot the $a_{0}^{\prime}$ and $a_{0}^{\prime \prime}$ contributions in the typical $B \rightarrow[K \bar{K} / \pi \eta] h$ decay in the second scenario of multiparticle configurations of $a_{0}$, as depicted in Fig. 3, where the top panel shows the result of channels $B^{+} \rightarrow a_{0}^{0}\left[\rightarrow K^{+} K^{-} / \pi^{0} \eta\right] \pi^{+} \quad$ (left) and $B^{+} \rightarrow$ $a_{0}^{\prime+}\left[\rightarrow K^{+} \bar{K}^{0} / \pi^{+} \eta\right] K^{0}$ (right) with the invariant mass starting from the thresholds and closing up at $3.0 \mathrm{GeV}$, the plots in the medium panel are depicted for the channels $B^{+} \rightarrow a_{0}^{\prime \prime 0}\left[\rightarrow K^{+} K^{-} / \pi^{0} \eta\right] \pi^{+}$(left) and $B^{+} \rightarrow$ $a_{0}^{\prime \prime+}\left[\rightarrow K^{+} \bar{K}^{0} / \pi^{+} \eta\right] K^{0}$ (right) with varying the invariant mass from thresholds to $4.0 \mathrm{GeV}$, and the bottom panel presents the result of channels $B^{+} \rightarrow a_{0}^{\prime \prime \prime \prime}\left[\rightarrow K^{+} \bar{K}^{0}\right] K^{0}$ (left) and $B^{+} \rightarrow a_{0}^{\prime / \prime \prime}\left[\rightarrow \pi^{+} \eta\right] K^{0}$ (right). We can easily get that (a) the contributions from $a_{0}^{\prime}$ in the channels $B^{+} \rightarrow$ $\left[\pi^{0} \eta / K^{+} K^{-}\right] \pi^{+}$and $B^{+} \rightarrow\left[\pi^{+} \eta / K^{+} \bar{K}^{0}\right] K^{0}$ in the second scenario of multiparticle configurations of $a_{0}$ are very close 


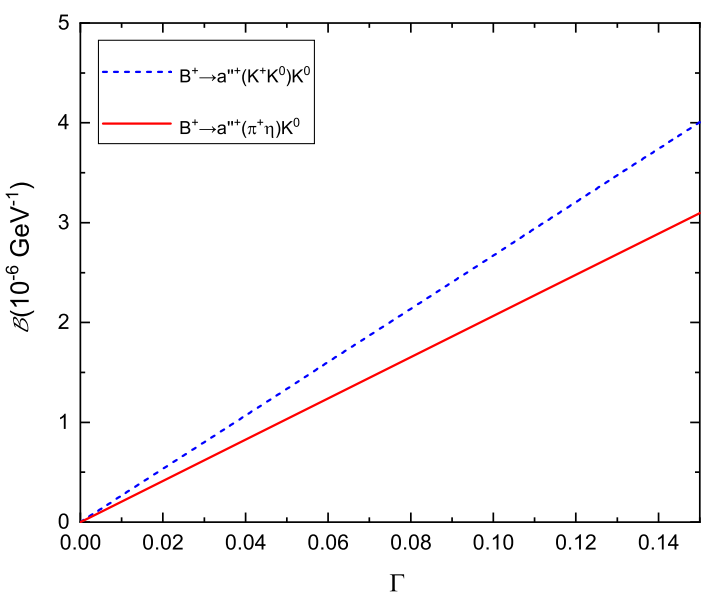

FIG. 4. Evolutions of $\mathcal{B}\left(B^{+} \rightarrow a_{0}^{\prime \prime+}\left[\rightarrow K^{+} \bar{K}^{0} / \pi^{+} \eta\right] K^{0}\right)$ on the partial widths $\Gamma_{a_{0}^{\prime \prime} \rightarrow K \bar{K} / \pi \eta}$ in the second scenario of multiparticle configurations of $a_{0}$ mesons.

to that obtained in the first scenario, we would like to mark again that the neutral $B$ meson decaying channels $B^{0} \rightarrow\left[K^{ \pm} K^{0} / \pi^{ \pm} \eta\right] \pi^{\mp}$, even though they have similar shapes, have apparent different predictions in magnitude in these two scenarios; (b) the contributions from $a_{0}^{\prime \prime}$ are larger than that from $a_{0}^{\prime}$, in the $\left[\pi^{0} \eta / K^{+} K^{-}\right] \pi^{+}$channels even larger by about an order. This is an impressive result, but it is not surprising if we look at the twist 2 LCDAs in Eq. (13) and the relevant parameters, and we look forward to the experiment check; (c) the $a_{0}^{\prime \prime}$ contributions in the channels $\left[\pi^{+} \eta\right] K^{0}$ and $\left[K^{+} \bar{K}^{0}\right] K^{0}$ are almost overlapping because the $a_{0}^{\prime \prime}$ is far away from the $K \bar{K}$ and $\pi \eta$ thresholds, as we can also find in the channels $\left[\pi^{0} \eta\right] \pi^{+}$and $[K \bar{K}] \pi^{+}$if we consider both the $K^{+} K^{-}$and $K^{0} \bar{K}^{0}$ contributions; (d) the partial widths of $a_{0}^{\prime \prime} \rightarrow K \bar{K} / \pi \eta$ effect are significant for the result of the quasitwo-body, we plot the varying band in the bottom panel by taking the result $\Gamma_{a_{0}^{\prime \prime}} \rightarrow K \bar{K}=94 \pm 54 \mathrm{MeV}$ and $\Gamma_{a_{0}^{\prime \prime}} \rightarrow$ $\pi \eta=94 \pm 16 \mathrm{MeV}$ obtained from the eLSM model [57]. We depict in Fig. 4 the dependence of the branching fractions of $B^{+} \rightarrow a_{0}^{\prime \prime+}\left[\rightarrow K^{+} \bar{K}^{0} / \pi^{+} \eta\right] K^{0}$ on the partial widths $\Gamma_{a_{0}^{\prime \prime} \rightarrow K \bar{K} / \pi \eta}$ with considering the largest uncertainties in Eq. (22). It is shown that the width effect of $a_{0}^{\prime \prime}$ in the relevant quasi-two-body $B$ decays is linear, so we suggest these channels in $B$ decays to determine the partial widths $\Gamma_{a_{0}^{\prime \prime} \rightarrow K \bar{K} / \pi \eta}$.

\section{CONCLUSION}

Motivated by the discrepancy between the experimental measurements of three-body $B \rightarrow a_{0}(980)[\rightarrow \pi \eta] K$ decays and the theoretical predictions of two-body $B \rightarrow a_{0}(980) K$ decays, we study the contributions from $a_{0}$ in the threebody $B \rightarrow[\pi \eta]([K \bar{K}]) h$ decays in the framework of the PQCD approach, where the width effects of the intermediated isovector scalar mesons $a_{0}$ are demonstrated in detail. This is also the first systematical study of the width effect in $B \rightarrow a_{0}$ decays. In the face of controversy for the multiparticle configurations of $a_{0}(980)$, particularly in the $B$ decays, we consider two scenarios where the first one states that $a_{0}(980)$ is the lowest-lying $q \bar{q}$ state, and the second one says that the lowest-lying $q \bar{q}$ state is $a_{0}(1450)$ while $a_{0}(980)$ is a compact tetraquark state or $K \bar{K}$ bound state.

We find that the width effect from intermediate $a_{0}$ states is significant in the relevant quasi-two-body decaying channels, with which we extract the branching fractions of corresponding two-body decays under narrow width approximation, showing a large difference to the previous direct two-body calculation under the static $a_{0}(980)$ assumption. Our calculations show that the $a_{0}(980)$ as the lowest-lying $q \bar{q}$ state cannot be ruled out in $B$ decays within acceptable limits with the current measurements. To examine the nature of $a_{0}$ state in $B$ decays, we suggest several channels for the future experiments. The first candidate is the $B \rightarrow a_{0}^{-}\left[\rightarrow \pi^{-} \eta\right] \pi^{+}$mode with the largest branching fraction from the calculation under the first scenario, the second ones are the $B^{0} \rightarrow$ $a_{0}^{ \pm}(1450)\left[\rightarrow K^{ \pm} \bar{K}^{0} / \pi^{ \pm} \pi^{0}\right] \pi^{\mp}$ modes, whose branching fractions obtained in the first scenario are about three times smaller in magnitude than that obtained in the second scenario. Last, but not the least, is the partial widths $\left(\Gamma_{a_{0}(1950) \rightarrow K \bar{K} / \pi \eta}\right)$ dependence of the partial branching fractions of $B \rightarrow a_{0}(1950)[K \bar{K} / \pi \eta] h$ modes. This dependence is shown in the linear behavior and could be examined by the future data. As a by-product, we present $a_{0}$ mesons contributions in the CKM suppressed $B_{s}$ decays, which seems harder for the near future experiments.

\section{ACKNOWLEDGMENTS}

We would like to thank Wen-fei Wang for proposing this project to us, and to Hai-yang Cheng for the fruitful discussion. This work is supported by the National Science Foundation of China (NSFC) under the Grants No. 11805060, No. 11975112, No. 11947011 and the Joint Large Scale Scientific Facility Funds of the NSFC and CAS under Contract No. U1932110. S. C. is also supported by the Natural Science Foundation of Hunan Province, China (Grant No. 2020JJ4160). A. J. M. is also supported by the Natural Science Foundation of Jiangsu Province, China (Grant No. BK20191010) and the Scientific Research Foundation of Nanjing Institute of Technology (Grant No. YKJ201854).

\section{APPENDIX A: PROBING $a_{0}$ MESONS IN THE QUASI-TWO-BODY $B_{s}$ DECAYS}

We also predict the contributions from isovector scalar mesons in the CKM suppressed $B_{s}$ decays under, as presented in Table VI and Table VII under scenarios I and II, respectively, the channel $\left(B_{s}^{0} \rightarrow a_{0}^{-}\left[\pi^{-} \eta\right] K^{+}\right)$with the predicted branching fraction $\left(0.75_{-0.14-0.12}^{+0.22+0.13}\right) \times 10^{-6}$ is the most possible available at the near future experiments. 
TABLE VI. The same as Table II, but for the $B_{s}^{0} \rightarrow a_{0}^{(\prime)}[\rightarrow K \bar{K} / \pi \eta] h$ decays.

\begin{tabular}{|c|c|c|c|}
\hline Decay modes & Quasi-two-body & Narrow approximation & $\mathrm{CPV}$ \\
\hline$B_{s}^{0} \rightarrow a_{0}^{+}\left[\rightarrow K^{+} \bar{K}^{0}\right] \pi^{-}$ & $0.03_{-0.00-0.01}^{+0.00+0.01}$ & & $-8.1_{-2.7-7.1}^{+6.0+4.8}$ \\
\hline$\rightarrow a_{0}^{+}\left[\rightarrow \pi^{+} \eta\right] \pi^{-}$ & $0.17_{-0.00-0.04}^{+0.00+0.01}$ & $0.25_{-0.01-0.05}^{+0.00+0.09}$ & $-11.7_{-0.5}^{+2.7+1.5}$ \\
\hline$B_{s}^{0} \rightarrow a_{0}^{0}\left[\rightarrow K^{-} K^{+}\right] \pi^{0}$ & $0.04_{-0.01-0.01}^{+0.00+0.01}$ & & $16.5_{-0.3-3.5}^{+2.5+8.0}$ \\
\hline$\rightarrow a_{0}^{0}\left[\rightarrow \pi^{0} \eta\right] \pi^{0}$ & $0.49^{+0.03+0.16}$ & $0.70_{-0.09+0.14}^{+0.04+0.24}$ & $22.7^{+1.2+2.6}$ \\
\hline$B_{s}^{0} \rightarrow a_{0}^{-}\left[\rightarrow K^{-} K^{0}\right] \pi^{+}$ & $0.03_{-0.01-0.00}^{+0.01+0.00}$ & & $22.1_{-3.1-10.8}^{+6.5+12.9}$ \\
\hline$\rightarrow a_{0}^{-}\left[\rightarrow \pi^{-} \eta\right] \pi^{+}$ & $0.14_{-0.02+0.09}^{+0.01+0.09}$ & $0.20_{-0.03}^{+0.08+0.04}$ & $44.4_{-9}^{+4.2+2.9}$ \\
\hline$B_{s}^{0} \rightarrow a_{0}^{0}\left[\rightarrow K^{-} K^{+}\right] K^{0}$ & $0.16_{-0.03-0.02}^{+0.05+0.02}$ & & $54.8_{-53-7.5}^{+0.1+6.4}$ \\
\hline$\rightarrow a_{0}^{0}\left[\rightarrow \pi^{0} \eta\right] K^{0}$ & $0.74_{-0.15-0.07}^{+0.19+0.09}$ & $1.05_{-0.21-0.10}^{+0.28+0.12}$ & $61.4_{-1.6-7.5}^{+1.3+5.9}$ \\
\hline$B_{s}^{0} \rightarrow a_{0}^{-}\left[\rightarrow K^{-} K^{0}\right] K^{+}$ & $0.07_{-0.01-0.01}^{+0.02+0.01}$ & & $81.5_{-8.8-3.5}^{+4.8+1.5}$ \\
\hline$\rightarrow a_{0}^{-}\left[\rightarrow \pi^{-} \eta\right] K^{+}$ & $0.75_{-0.13-0.12}^{+0.22+0.14}$ & $1.06_{-0.19-0.18}^{+0.32+0.20}$ & $77.8_{-9.1-3.1}^{+1.6+5.6}$ \\
\hline$B_{s}^{0} \rightarrow a_{0}^{\prime+}\left[\rightarrow K^{+} \bar{K}^{0}\right] \pi^{-}$ & $0.09_{-0.02-0.02}^{+0.02+0.03}$ & $1.05_{-0.21-0.28}^{+0.29+0.33}$ & $-10.1_{-0.8-0.3}^{+0.2+1.8}$ \\
\hline$\rightarrow a_{0}^{\prime+}\left[\rightarrow \pi^{+} \eta\right] \pi^{-}$ & $0.10_{-0.02-0.03}^{+0.03+0.03}$ & $1.08_{-0.22-0.31}^{+0.31+0.33}$ & $-8.3_{-3.0-3.9}^{+1.8+1.8}$ \\
\hline$B_{s}^{0} \rightarrow a_{0}^{\prime 0}\left[\rightarrow K^{-} K^{+}\right] \pi^{0}$ & $0.13_{-0.02-0.03}^{+0.03+0.03}$ & $3.07_{-0.06-0.71}^{+0.61+0.84}$ & $19.7_{-04-3.8}^{+1.9+2.0}$ \\
\hline$\rightarrow a_{0}^{\prime 0}\left[\rightarrow \pi^{0} \eta\right] \pi^{0}$ & $0.30_{-0.06-0.07}^{+0.06+0.08}$ & $3.20_{-0.61-0.71}^{+0.68+0.84}$ & $20.8_{-0.2-3.9}^{+1.2+2.0}$ \\
\hline$B_{s}^{0} \rightarrow a_{0}^{\prime-}\left[\rightarrow K^{-} K^{0}\right] \pi^{+}$ & $0.05_{-0.01-0.01}^{+0.01+0.02}$ & $0.67_{-0.12-0.18}^{+0.16+0.22}$ & $53.9_{-3.8-3.5}^{+0.5+1.5}$ \\
\hline$\rightarrow a_{0}^{\prime-}\left[\rightarrow \pi^{-} \eta\right] \pi^{+}$ & $0.06_{-0.01-0.02}^{+0.02+0.02}$ & $0.69_{-0.13-0.18}^{+0.18+0.21}$ & $55.4_{-2.0-2.5}^{+0.5+2.7}$ \\
\hline$B_{s}^{0} \rightarrow a_{0}^{\prime 0}\left[\rightarrow K^{-} K^{+}\right] K^{0}$ & $0.07_{-0.01-0.02}^{+0.01+0.02}$ & $0.88_{-0.09-0.29}^{+0.12+0.29}$ & $-6.6_{-5.0-12.1}^{+0.9+9.2}$ \\
\hline$\rightarrow a_{0}^{\prime 0}\left[\rightarrow \pi^{0} \eta\right] K^{0}$ & $0.08_{-0.01-0.02}^{+0.01+0.03}$ & $0.89_{-0.08-0.28}^{+0.13+0.30}$ & $-8.8_{-5.9-12.1}^{+2.2+10.4}$ \\
\hline$B_{s}^{0} \rightarrow a_{0}^{\prime-}\left[\rightarrow K^{-} K^{0}\right] K^{+}$ & $0.03_{-0.00-0.01}^{+0.01+0.01}$ & $0.64_{-0.04-0.12}^{+0.11+0.25}$ & $-28.3_{-9.1-8.6}^{+2.7+10.1}$ \\
\hline$\rightarrow a_{0}^{\prime-}\left[\rightarrow \pi^{-} \eta\right] K^{+}$ & $0.06_{-0.00-0.01}^{+0.01+0.02}$ & $0.66_{-0.04-0.13}^{+0.11+0.23}$ & $-27.8_{-6.1-8.6}^{+1.4+6.1}$ \\
\hline
\end{tabular}

TABLE VII. The same as Table IV, but for the $B_{s}^{0} \rightarrow a_{0}^{\prime / \prime \prime}[\rightarrow K \bar{K} / \pi \eta] h$ decays.

\begin{tabular}{|c|c|c|c|}
\hline Decay modes & Quasi-two-body & Narrow approximation & $\mathrm{CPV}$ \\
\hline$B_{s}^{0} \rightarrow a_{0}^{++}\left[\rightarrow K^{+} \bar{K}^{0}\right] \pi^{-}$ & $0.08_{-0.01-0.03}^{+0.02+0.03}$ & $1.02_{-0.18-0.35}^{+0.20+0.39}$ & $-0.9_{-0.2-0.7}^{+1.0+1.0}$ \\
\hline$\rightarrow a_{0}^{\prime+}\left[\rightarrow \pi^{+} \eta\right] \pi^{-}$ & $0.10_{-0.02-0.03}^{+0.02+0.03}$ & $1.05_{-0.19-0.36}^{+0.18+0.40}$ & $-0.9_{-0.3-0.3}^{+1.6+0.3}$ \\
\hline$B_{s}^{0} \rightarrow a_{0}^{\prime 0}\left[\rightarrow K^{-} K^{+}\right] \pi^{0}$ & $0.11_{-0.02-0.04}^{+0.02+0.04}$ & $2.73_{-0.49-0.92}^{+0.58+1.11}$ & $17.9_{-1.3-2.3}^{+0.3+1.5}$ \\
\hline$\rightarrow a_{0}^{\prime 0}\left[\rightarrow \pi^{0} \eta\right] \pi^{0}$ & $0.26_{-0.05-0.08}^{+0.06+0.10}$ & $2.79_{-0.50-0.92}^{+0.50+1.14}$ & $16.2_{-0.1-1.0}^{+0.6+0.8}$ \\
\hline$B_{s}^{0} \rightarrow a_{0}^{--}\left[\rightarrow K^{-} K^{0}\right] \pi^{+}$ & $0.03_{-0.01-0.01}^{+0.01+0.02}$ & $0.36_{-0.06-0.17}^{+0.07+0.14}$ & $26.8_{-8.5-6.5}^{+4.8+4.8}$ \\
\hline$\rightarrow a_{0}^{\prime-}\left[\rightarrow \pi^{-} \eta\right] \pi^{+}$ & $0.03_{-0.01-0.02}^{+0.01+0.02}$ & $0.36_{-0.05-0.16}^{+0.09+0.25}$ & $22.4_{-2.2-2.2}^{+6.7+1.5}$ \\
\hline$B_{s}^{0} \rightarrow a_{0}^{\prime 0}\left[\rightarrow K^{-} K^{+}\right] K^{0}$ & $0.15_{-0.02-0.03}^{+0.04+0.04}$ & $1.88_{-0.28-0.33}^{+0.04+0.39}$ & $22.6_{-1.6-3.5}^{+2.2+5.1}$ \\
\hline$\rightarrow a_{0}^{\prime 0}\left[\rightarrow \pi^{0} \eta\right] K^{0}$ & $0.17_{-0.03-0.03}^{+0.04+0.04}$ & $1.88_{-0.27-0.31}^{+0.44+0.40}$ & $23.4_{-1.9-4.2}^{+2.2+4.4}$ \\
\hline$B_{s}^{0} \rightarrow a_{0}^{\prime-}\left[\rightarrow K^{-} K^{0}\right] K^{+}$ & $0.04_{-0.01-0.01}^{+0.01+0.01}$ & $1.07_{-0.16-0.19}^{+0.27+0.23}$ & $57.1_{-1.4-5.4}^{+0.1+6.1}$ \\
\hline$\rightarrow a_{0}^{\prime-}\left[\rightarrow \pi^{-} \eta\right] K^{+}$ & $0.10_{-0.01-0.01}^{+0.02+0.03}$ & $1.09_{-0.16-0.19}^{+0.10-0.19}$ & $57.6_{-0.9-4.8}^{+0.2+7.1}$ \\
\hline$B_{s}^{0} \rightarrow a_{0}^{\prime \prime+}\left[\rightarrow K^{+} \bar{K}^{0}\right] \pi^{-}$ & $0.02_{-0.01-0.02}^{+0.01+0.06} \pm 0.01$ & $0.07_{-0.01-0.06}^{+0.03+17}$ & $50.9_{-9.3-11.0}^{+6.0+13.3}$ \\
\hline$\rightarrow a_{0}^{\prime \prime+}\left[\rightarrow \pi^{+} \eta\right] \pi^{-}$ & $0.03_{-0.01-0.03}^{+0.01+0.06} \pm 0.01$ & $0.08_{-0.02-0.07}^{+0.02+0.00}$ & $48.5_{-9.6-10.5}^{+3.7+8.6}$ \\
\hline$B_{s}^{0} \rightarrow a_{0}^{\prime \prime 0}\left[\rightarrow K^{-} K^{+}\right] \pi^{0}$ & $0.03_{-0.0-0.04}^{+0.01+0.08} \pm 0.02$ & $0.20_{-0.01-0.17}^{+0.08+0.48}$ & $37.0_{-0.9-11.1}^{+3.8+6.0}$ \\
\hline$\rightarrow a_{0}^{\prime \prime 0}\left[\rightarrow \pi^{0} \eta\right] \pi^{0}$ & $0.07_{-0.01-0.07}^{+0.02+0.16} \pm 0.01$ & $0.22_{-0.03-0.19}^{+0.05+0.48}$ & $42.7_{-3.7-5.8}^{+4.1+13.2}$ \\
\hline$B_{s}^{0} \rightarrow a_{0}^{\prime \prime-}\left[\rightarrow K^{-} K^{0}\right] \pi^{+}$ & $0.10_{-0.01-0.05}^{+0.01+0.08} \pm 0.06$ & $0.30_{-0.04-0.17}^{+0.03+0.24}$ & $35.3_{-0.4-9.2}^{+2.0+13.7}$ \\
\hline$\rightarrow a_{0}^{\prime \prime-}\left[\rightarrow \pi^{-} \eta\right] \pi^{+}$ & $0.10_{-0.02-0.06}^{+0.01+0.08} \pm 0.02$ & $0.31_{-0.05-0.18}^{+0.03+0.23}$ & $31.0_{-0.5-7.5}^{+3.4+8.7}$ \\
\hline$B_{s}^{0} \rightarrow a_{0}^{\prime \prime 0}\left[\rightarrow K^{-} K^{+}\right] K^{0}$ & $0.69_{-0.16-0.18}^{+0.24+0.24} \pm 0.39$ & $2.06_{-0.48-0.56}^{+0.71+0.74}$ & $-3.6_{-3.5-8.8}^{+3.4+7.5}$ \\
\hline$\rightarrow a_{0}^{\prime \prime 0}\left[\rightarrow \pi^{0} \eta\right] K^{0}$ & $0.69_{-0.16-0.19}^{+0.24+0.25} \pm 0.12$ & $2.06_{-0.48-0.57}^{+0.71+0.74}$ & $-3.8_{-3.6-9.3}^{+3.4+7.5}$ \\
\hline$B_{s}^{0} \rightarrow a_{0}^{\prime \prime-}\left[\rightarrow K^{-} K^{0}\right] K^{+}$ & $0.20_{-0.05-0.05}^{+0.07+0.08} \pm 0.11$ & $1.19_{-0.28-0.33}^{+0.41+0.47}$ & $-6.6_{-8.9-10.9}^{+8.8+10.6}$ \\
\hline$\rightarrow a_{0}^{\prime \prime-}\left[\rightarrow \pi^{-} \eta\right] K^{+}$ & $0.40_{-0.09-0.10}^{+0.14+0.16} \pm 0.07$ & $1.18_{-0.27-0.32}^{+0.43+0.50}$ & $-7.6_{-7.7-10.3}^{+9.9+9.5}$ \\
\hline
\end{tabular}




\section{APPENDIX B: DECAY AMPLITUDES}

In this section, we list the Lorentz invariant decay amplitude $\mathcal{A}$ for the considered quasi-two-body decay in the PQCD approach.

$$
\begin{aligned}
& \mathcal{A}\left(B^{+} \rightarrow a_{0}^{+} \pi^{0}\right)=\frac{G_{F}}{2}\left\{V _ { u b } ^ { * } V _ { u d } \left[\left(a_{1}\left(F_{T h}^{L L}+F_{A h}^{L L}-F_{A a_{0}}^{L L}\right)+a_{2} F_{T a_{0}}^{L L}+C_{1}\left(M_{T h}^{L L}+M_{A h}^{L L}-M_{A a_{0}}^{L L}\right)\right.\right.\right. \\
& \left.+C_{2} M_{T a_{0}}^{L L}\right]-V_{t b}^{*} V_{t d}\left[\left(-a_{4}+\frac{5 C_{9}}{3}+C_{10}-\frac{3 a_{7}}{2}\right) F_{T a_{0}}^{L L}-\left(a_{6}-\frac{a_{8}}{2}\right) F_{T a_{0}}^{S P}\right. \\
& +\left(\frac{C_{9}+3 C_{10}}{2}-C_{3}\right) M_{T a_{0}}^{L L}-\left(C_{5}-\frac{C_{7}}{2}\right) M_{T a_{0}}^{L R}+\frac{3 C_{8}}{2} M_{T a_{0}}^{S P} \\
& +\left(a_{4}+a_{10}\right)\left(F_{T h}^{L L}+F_{A h}^{L L}-F_{A a_{0}}^{L L}\right)+\left(a_{6}+a_{8}\right)\left(F_{T h}^{S P}+F_{A h}^{S P}-F_{A a_{0}}^{S P}\right) \\
& \left.\left.+\left(C_{3}+C_{9}\right)\left(M_{T h}^{L L}+M_{A h}^{L L}-M_{A a_{0}}^{L L}\right)+\left(C_{5}+C_{7}\right)\left(M_{T h}^{L R}+M_{A h}^{L R}-M_{A a_{0}}^{L R}\right)\right]\right\}, \\
& \mathcal{A}\left(B^{+} \rightarrow a_{0}^{0} \pi^{+}\right)=\frac{G_{F}}{2}\left\{V _ { u b } ^ { * } V _ { u d } \left[a_{1}\left(F_{T a_{0}}^{L L}+F_{A a_{0}}^{L L}-F_{A h}^{L L}\right)+a_{2} F_{T h}^{L L}+C_{1}\left(M_{T a_{0}}^{L L}+M_{A a_{0}}^{L L}-M_{A h}^{L L}\right)\right.\right. \\
& \left.+C_{2} M_{T h}^{L L}\right]-V_{t b}^{*} V_{t d}\left[\left(a_{4}+a_{10}\right)\left(F_{T a_{0}}^{L L}+F_{A a_{0}}^{L L}-F_{A h}^{L L}\right)-\left(a_{6}-\frac{a_{8}}{2}\right) F_{T h}^{S P}\right. \\
& +\left(a_{6}+a_{8}\right)\left(F_{T a_{0}}^{S P}+F_{A a_{0}}^{S P}-F_{A h}^{S P}\right)+\left(C_{3}+C_{9}\right)\left(M_{T a_{0}}^{L L}+M_{A a_{0}}^{L L}-M_{A h}^{L L}\right) \\
& +\left(C_{5}+C_{7}\right)\left(M_{T a_{0}}^{L R}+M_{A a_{0}}^{L R}-M_{T h}^{L R}\right)+\left(\frac{5}{3} C_{9}+C_{10}+\frac{3 a_{7}}{2}-a_{4}\right) F_{T h}^{L L} \\
& \left.\left.+\left(\frac{C_{9}+3 C_{10}}{2}-C_{3}\right) M_{T h}^{L L}-\left(C_{5}-\frac{C_{7}}{2}\right) M_{T h}^{L R}+\frac{3 C_{8}}{2} M_{T h}^{S P}\right]\right\}, \\
& \mathcal{A}\left(B^{+} \rightarrow a_{0}^{+} K^{0}\right)=\frac{G_{F}}{\sqrt{2}}\left\{V_{u b}^{*} V_{u s}\left[a_{1} F_{A a_{0}}^{L L}+C_{1} M_{A a_{0}}^{L L}\right]-V_{t b}^{*} V_{t s}\left[\left(a_{4}-\frac{a_{10}}{2}\right) F_{T a_{0}}^{L L}\right.\right. \\
& +\left(a_{6}-\frac{a_{8}}{2}\right) F_{T a_{0}}^{S P}+\left(C_{3}-\frac{C_{9}}{2}\right) M_{T a_{0}}^{L L}+\left(C_{5}-\frac{C_{7}}{2}\right) M_{T a_{0}}^{L R}+\left(a_{4}+a_{10}\right) F_{A a_{0}}^{L L} \\
& \left.\left.+\left(C_{3}+C_{9}\right) M_{A a_{0}}^{L L}+\left(a_{6}+a_{8}\right) F_{A a_{0}}^{S P}+\left(C_{5}+C_{7}\right) M_{A a_{0}}^{L R}\right]\right\} \text {, } \\
& \mathcal{A}\left(B^{+} \rightarrow a_{0}^{0} K^{+}\right)=\frac{G_{F}}{2}\left\{V _ { u b } ^ { * } V _ { u s } \left[a_{1}\left(F_{T a_{0}}^{L L}+F_{A a_{0}}^{L L}\right)+a_{2} F_{T h}^{L L}+C_{1}\left(M_{T a_{0}}^{L L}+M_{A a_{0}}^{L L}\right)\right.\right. \\
& \left.+C_{2} M_{T h}^{L L}\right]-V_{t b}^{*} V_{t s}\left[\left(a_{4}+a_{10}\right)\left(F_{T a_{0}}^{L L}+F_{A a_{0}}^{L L}\right)+\left(a_{6}+a_{8}\right)\left(F_{T a_{0}}^{S P}+F_{A a_{0}}^{S P}\right)\right. \\
& +\left(C_{3}+C_{9}\right)\left(M_{T a_{0}}^{L L}+M_{A a_{0}}^{L L}\right)+\left(C_{5}+C_{7}\right)\left(M_{T a_{0}}^{L R}+M_{A a_{0}}^{L R}\right) \\
& \left.\left.+\frac{3}{2}\left(a_{7}+a_{9}\right) F_{T h}^{L L}+\frac{3 C_{10}}{2} M_{T h}^{L L}+\frac{3 C_{8}}{2} M_{T h}^{S P}\right]\right\},
\end{aligned}
$$




$$
\begin{aligned}
& \mathcal{A}\left(B^{0} \rightarrow a_{0}^{+} \pi^{-}\right)=\frac{G_{F}}{\sqrt{2}}\left\{V_{u b}^{*} V_{u d}\left[a_{2} F_{A a_{0}}^{L L}+C_{2} M_{A a_{0}}^{L L}+a_{1} F_{T h}^{L L}+C_{1} M_{T h}^{L L}\right]\right. \\
& -V_{t b}^{*} V_{t d}\left[\left(a_{3}+a_{9}-a_{5}-a_{7}\right) F_{A a_{0}}^{L L}+\left(C_{4}+C_{10}\right) M_{A a_{0}}^{L L}\right. \\
& +\left(C_{6}+C_{8}\right) M_{A a_{0}}^{S P}+\left(a_{4}+a_{10}\right) F_{T h}^{L L}+\left(a_{6}+a_{8}\right) F_{T h}^{S P} \\
& +\left(C_{3}+C_{9}\right) M_{T h}^{L L}+\left(C_{5}+C_{7}\right) M_{T h}^{L R}+\left(\frac{4}{3}\left(C_{3}+C_{4}-\frac{C_{9}}{2}-\frac{C_{10}}{2}\right)\right. \\
& \left.-a_{5}+\frac{a_{7}}{2}\right) F_{A h}^{L L}+\left(a_{6}-\frac{a_{8}}{2}\right) F_{A h}^{S P}+\left(C_{3}+C_{4}-\frac{C_{9}}{2}-\frac{C_{10}}{2}\right) M_{A h}^{L L} \\
& \left.\left.+\left(C_{5}-\frac{C_{7}}{2}\right) M_{A h}^{L R}+\left(C_{6}-\frac{C_{8}}{2}\right) M_{A h}^{S P}\right]\right\} \\
& \mathcal{A}\left(B^{0} \rightarrow a_{0}^{0} \pi^{0}\right)=\frac{G_{F}}{2 \sqrt{2}}\left\{V _ { u b } ^ { * } V _ { u d } \left[a_{2}\left(F_{A a_{0}}^{L L}+F_{A h}^{L L}-F_{T a_{0}}^{L L}-F_{T h}^{L L}\right)+C_{2}\left(M_{A a_{0}}^{L L}+M_{A h}^{L L}\right.\right.\right. \\
& \left.\left.-M_{T a_{0}}^{L L}-M_{T h}^{L L}\right)\right]-V_{t b}^{*} V_{t d}\left[\left(a_{4}-\frac{5 C_{9}}{3}-C_{10}+\frac{3 a_{7}}{2}\right) F_{T a_{0}}^{L L}\right. \\
& +\left(a_{6}-\frac{a_{8}}{2}\right)\left(F_{T a_{0}}^{S P}+F_{A a_{0}}^{S P}+F_{T h}^{S P}+F_{A h}^{S P}\right)+\left(C_{3}-\frac{C_{9}+3 C_{10}}{2}\right)\left(M_{T a_{0}}^{L L}+M_{T h}^{L L}\right) \\
& +\left(C_{5}-\frac{C_{7}}{2}\right)\left(M_{T a_{0}}^{L R}+M_{A a_{0}}^{L R}+M_{T h}^{L R}+M_{A h}^{L R}\right)-\frac{3 C_{8}}{2}\left(M_{T a_{0}}^{S P}+M_{T h}^{S P}\right) \\
& +\left(\frac{7 C_{3}+5 C_{4}+C_{9}-C_{10}}{3}-2 a_{5}-\frac{a_{7}}{2}\right)\left(F_{A a_{0}}^{L L}+F_{A h}^{L L}\right) \\
& +\left(C_{3}+2 C_{4}-\frac{C_{9}-C_{10}}{2}\right)\left(M_{A a_{0}}^{L L}+M_{A h}^{L L}\right)+\left(2 C_{6}+\frac{C_{8}}{2}\right)\left(M_{A a_{0}}^{S P}+M_{A h}^{S P}\right) \\
& \left.+\left(a_{4}-\frac{5 C_{9}}{3}-C_{10}-\frac{3 a_{7}}{2}\right) F_{T h}^{L L}\right] \\
& \mathcal{A}\left(B^{0} \rightarrow a_{0}^{-} \pi^{+}\right)=\frac{G_{F}}{\sqrt{2}}\left\{V_{u b}^{*} V_{u d}\left[a_{1} F_{T a_{0}}^{L L}+a_{2} F_{A h}^{L L}+C_{1} M_{T a_{0}}^{L L}+C_{2} M_{A h}^{L L}\right]-V_{t b}^{*} V_{t d}\left[\left(a_{4}\right.\right.\right. \\
& \left.+a_{10}\right) F_{T a_{0}}^{L L}+\left(a_{6}+a_{8}\right) F_{T a_{0}}^{S P}+\left(C_{3}+C_{9}\right) M_{T a_{0}}^{L L}+\left(C_{5}+C_{7}\right) M_{T a_{0}}^{L R} \\
& +\left(\frac{4}{3}\left(C_{3}+C_{4}-\frac{C_{9}+C_{10}}{2}\right)-a_{5}+\frac{a_{7}}{2}\right) F_{A a_{0}}^{L L}+\left(a_{6}-\frac{a_{8}}{2}\right) F_{A a_{0}}^{S P} \\
& +\left(C_{3}+C_{4}-\frac{C_{9}+C_{10}}{2}\right) M_{A a_{0}}^{L L}+\left(C_{5}-\frac{C_{7}}{2}\right) M_{A a_{0}}^{L R}+\left(C_{6}-\frac{C_{8}}{2}\right) M_{A a_{0}}^{S P} \\
& \left.\left.+\left(a_{3}+a_{9}-a_{5}-a_{7}\right) F_{A h}^{L L}+\left(C_{4}+C_{10}\right) M_{A h}^{L L}+\left(C_{6}+C_{8}\right) M_{A h}^{S P}\right]\right\} \text {, } \\
& \mathcal{A}\left(B^{0} \rightarrow a_{0}^{0} K^{0}\right)=\frac{G_{F}}{2}\left\{V_{u b}^{*} V_{u s}\left[a_{2} F_{T h}^{L L}+C_{2} M_{T h}^{L L}\right]-V_{t b}^{*} V_{t s}\left[-\left(a_{4}-\frac{a_{10}}{2}\right)\left(F_{T a_{0}}^{L L}+F_{A a_{0}}^{L L}\right)\right.\right. \\
& -\left(a_{6}-\frac{a_{8}}{2}\right)\left(F_{T a_{0}}^{S P}+F_{A a_{0}}^{S P}\right)-\left(C_{3}-\frac{C_{9}}{2}\right)\left(M_{T a_{0}}^{L L}+M_{A a_{0}}^{L L}\right) \\
& -\left(C_{5}-\frac{C_{7}}{2}\right)\left(M_{T a_{0}}^{L R}+M_{A a_{0}}^{L R}\right)+\frac{3}{2}\left(a_{7}+a_{9}\right) F_{T h}^{L L}+\frac{3 C_{10}}{2} M_{T h}^{L L} \\
& \left.\left.+\frac{3 C_{8}}{2} M_{T h}^{S P}\right]\right\} \text {, }
\end{aligned}
$$




$$
\begin{aligned}
& \mathcal{A}\left(B^{0} \rightarrow a_{0}^{-} K^{+}\right)=\frac{G_{F}}{\sqrt{2}}\left\{V_{u b}^{*} V_{u s}\left[a_{1} F_{T a_{0}}^{L L}+C_{1} M_{T a_{0}}^{L L}\right]-V_{t b}^{*} V_{t s}\left[\left(a_{4}+a_{10}\right) F_{T a_{0}}^{L L}\right.\right. \\
& +\left(a_{6}+a_{8}\right) F_{T a_{0}}^{S P}+\left(C_{3}+C_{9}\right) M_{T a_{0}}^{L L}+\left(C_{5}+C_{7}\right) M_{T a_{0}}^{L R} \\
& +\left(a_{4}-\frac{a_{10}}{2}\right) F_{A a_{0}}^{L L}+\left(a_{6}-\frac{a_{8}}{2}\right) F_{A a_{0}}^{S P}+\left(C_{3}-\frac{C_{9}}{2}\right) M_{A a_{0}}^{L L} \\
& \left.\left.+\left(C_{5}-\frac{C_{7}}{2}\right) M_{A a_{0}}^{L R}\right]\right\} \\
& \mathcal{A}\left(B_{s}^{0} \rightarrow a_{0}^{+} \pi^{-}\right)=\frac{G_{F}}{\sqrt{2}}\left\{V_{u b}^{*} V_{u s}\left[a_{2} F_{A a_{0}}^{L L}+C_{2} M_{A a_{0}}^{L L}\right]-V_{t b}^{*} V_{t s}\left[\left(a_{3}+a_{9}-a_{5}-a_{7}\right) F_{A a_{0}}^{L L}\right.\right. \\
& +\left(C_{4}+C_{10}\right) M_{A a_{0}}^{L L}+\left(C_{6}+C_{8}\right) M_{A a_{0}}^{S P}+\left(a_{3}-\frac{a_{9}}{2}-a_{5}+\frac{a_{7}}{2}\right) F_{A h}^{L L} \\
& \left.\left.+\left(C_{4}-\frac{C_{10}}{2}\right) M_{A h}^{L L}+\left(C_{6}-\frac{C_{8}}{2}\right) M_{A h}^{S P}\right]\right\} \\
& \mathcal{A}\left(B_{s}^{0} \rightarrow a_{0}^{0} \pi^{0}\right)=\frac{G_{F}}{2 \sqrt{2}}\left\{V_{u b}^{*} V_{u s}\left[a_{2}\left(F_{A a_{0}}^{L L}+F_{A h}^{L L}\right)+C_{2}\left(M_{A a_{0}}^{L L}+M_{A h}^{L L}\right)\right]\right. \\
& -V_{t b}^{*} V_{t s}\left[\left(2 a_{3}+\frac{a_{9}}{2}-2 a_{5}-\frac{a_{7}}{2}\right)\left(F_{A a_{0}}^{L L}+F_{A h}^{L L}\right)\right. \\
& \left.\left.+\left(2 C_{4}+\frac{C_{10}}{2}\right)\left(M_{A a_{0}}^{L L}+M_{A h}^{L L}\right)+\left(2 C_{6}+\frac{C_{8}}{2}\right)\left(M_{A a_{0}}^{S P}+M_{A h}^{S P}\right)\right]\right\} \\
& \mathcal{A}\left(B_{s}^{0} \rightarrow a_{0}^{-} \pi^{+}\right)=\frac{G_{F}}{\sqrt{2}}\left\{V_{u b}^{*} V_{u s}\left[a_{2} F_{A h}^{L L}+C_{2} M_{A h}^{L L}\right]-V_{t b}^{*} V_{t s}\left[\left(a_{3}-\frac{a_{9}}{2}-a_{5}+\frac{a_{7}}{2}\right) F_{A a_{0}}^{L L}\right.\right. \\
& +\left(C_{4}-\frac{C_{10}}{2}\right) M_{A a_{0}}^{L L}+\left(C_{6}-\frac{C_{8}}{2}\right) M_{A a_{0}}^{S P}+\left(a_{3}+a_{9}-a_{5}-a_{7}\right) F_{A h}^{L L} \\
& \left.\left.+\left(C_{4}+C_{10}\right) M_{A h}^{L L}+\left(C_{6}+C_{8}\right) M_{A h}^{S P}\right]\right\} \\
& \mathcal{A}\left(B_{s}^{0} \rightarrow a_{0}^{+} K^{-}\right)=\frac{G_{F}}{\sqrt{2}}\left\{V_{u b}^{*} V_{u d}\left[a_{1} F_{T h}^{L L}+C_{1} M_{T h}^{L L}\right]-V_{t b}^{*} V_{t d}\left[\left(a_{4}+a_{10}\right) F_{T h}^{L L}\right.\right. \\
& +\left(a_{6}+a_{8}\right) F_{T h}^{S P}+\left(C_{3}+C_{9}\right) M_{T h}^{L L}+\left(C_{5}+C_{7}\right) M_{T h}^{L R}+\left(a_{4}-\frac{a_{10}}{2}\right) F_{A h}^{L L} \\
& \left.\left.+\left(a_{6}-\frac{a_{8}}{2}\right) F_{A h}^{S P}+\left(C_{3}-\frac{C_{9}}{2}\right) M_{A h}^{L L}+\left(C_{5}-\frac{C_{7}}{2}\right) M_{A h}^{L R}\right]\right\} \\
& \mathcal{A}\left(B_{s}^{0} \rightarrow a_{0}^{0} \bar{K}^{0}\right)=\frac{G_{F}}{2}\left\{V_{u b}^{*} V_{u d}\left[a_{2} F_{T h}^{L L}+C_{2} M_{T h}^{L L}\right]-V_{t b}^{*} V_{t d}\left[\left(\frac{5 C_{9}}{3}+C_{10}+\frac{3 a_{7}}{2}-a_{4}\right) F_{T h}^{L L}\right.\right. \\
& -\left(a_{6}-\frac{a_{8}}{2}\right)\left(F_{T h}^{S P}+F_{A h}^{S P}\right)+\left(\frac{C_{9}}{2}+\frac{3 C_{10}}{2}-C_{3}\right) M_{T h}^{L L}-\left(C_{5}-\frac{C_{7}}{2}\right)\left(M_{T h}^{L R}+M_{A h}^{L R}\right) \\
& \left.\left.+\frac{3 C_{8}}{2} M_{T h}^{S P}-\left(a_{4}-\frac{a_{10}}{2}\right) F_{A h}^{L L}-\left(C_{3}-\frac{C_{9}}{2}\right) M_{A h}^{L L}\right]\right\}
\end{aligned}
$$

In these expressions, $G_{F}$ is the fermi coupling constant, $V$ 's are the CKM matrix elements, the combined Wilson coefficients $a_{i}$ are defined as 


$$
\begin{aligned}
& a_{1}=C_{2}+\frac{C_{1}}{3}, \quad a_{2}=C_{1}+\frac{C_{2}}{3}, \\
& a_{i}=C_{i}+\frac{C_{i+1}}{3} \quad \text { with } \quad i=3-10 .
\end{aligned}
$$

The factorizable and nonfactorizable amplitudes, $F$ and $M$, respectively, can be found in Refs. [38].

[1] R. L. Jaffe, Phys. Rev. D 15, 267 (1977); 15, 281 (1977).

[2] F. E. Close and N. A. Tornqvist, J. Phys. G 28, R249 (2002).

[3] N. N. Achasov and A. V. Kiselev, Phys. Rev. D 73, 054029 (2006); 74, 059902(E) (2006).

[4] N. N. Achasov and A. V. Kiselev, Phys. Rev. D 83, 054008 (2011).

[5] J. D. Weinstein and N. Isgur, Phys. Rev. Lett. 48, 659 (1982).

[6] J. D. Weinstein and N. Isgur, Phys. Rev. D 27, 588 (1983).

[7] S. Agaev, K. Azizi, and H. Sundu, Phys. Lett. B 781, 279 (2018).

[8] M. G. Alford and R. L. Jaffe, Nucl. Phys. B578, 367 (2000).

[9] L. Maiani, F. Piccinini, A. D. Polosa, and V. Riquer, Phys. Rev. Lett. 93, 212002 (2004).

[10] L. Maiani, A. D. Polosa, and V. Riquer, Phys. Lett. B 651, 129 (2007).

[11] J. D. Weinstein and N. Isgur, Phys. Rev. D 41, 2236 (1990).

[12] H. Y. Cheng, C. K. Chua, and K. C. Yang, Phys. Rev. D 73, 014017 (2006).

[13] S. Cheng and J. M. Shen, Eur. Phys. J. C 80, 554 (2020).

[14] Y. L. Shen, W. Wang, J. Zhu, and C. D. Lu, Eur. Phys. J. C 50, 877 (2007).

[15] Y. Li, D. C. Yan, J. Hua, Z. Rui, and H. n. Li, Phys. Rev. D 104, 096014 (2021).

[16] Z. Rui, Y. Li, and H. N. Li, J. High Energy Phys. 05 (2021) 082.

[17] W. F. Wang, Phys. Rev. D 103, 056021 (2021).

[18] J. Chai, S. Cheng, and W. F. Wang, Phys. Rev. D 103, 096016 (2021).

[19] R. Klein, T. Mannel, J. Virto, and K. K. Vos, J. High Energy Phys. 10 (2017) 117.

[20] T. Mannel, K. Olschewsky, and K. K. Vos, J. High Energy Phys. 06 (2020) 073.

[21] T. Huber, J. Virto, and K. K. Vos, J. High Energy Phys. 11 (2020) 103.

[22] Z. H. Zhang, Phys. Lett. B 820, 136537 (2021).

[23] H. Y. Cheng, C. W. Chiang, and C. K. Chua, Phys. Rev. D 103, 036017 (2021).

[24] H. Y. Cheng, C. W. Chiang, and Z. Q. Zhang, arXiv:2201 .00460 .

[25] S. Cheng, A. Khodjamirian, and J. Virto, J. High Energy Phys. 05 (2017) 157.

[26] C. Hambrock and A. Khodjamirian, Nucl. Phys. B905, 373 (2016).

[27] S. Cheng, A. Khodjamirian, and J. Virto, Phys. Rev. D 96, 051901 (2017).
[28] S. Descotes-Genon, A. Khodjamirian, and J. Virto, J. High Energy Phys. 12 (2019) 083.

[29] J. P. Lees et al. (BABAR Collaboration), Phys. Rev. D 93, 012005 (2016).

[30] P. Rubin et al. (CLEO Collaboration), Phys. Rev. D 78, 072003 (2008).

[31] R. Aaij et al. (LHCb Collaboration), Phys. Rev. D 93, 052018 (2016).

[32] C. Amsler et al. (Crystal Barrel Collaboration), Phys. Lett. B 333, 277 (1994).

[33] C. Amsler et al. (Crystal Barrel Collaboration), Phys. Lett. B 355, 425 (1995).

[34] M. V. Polyakov, Nucl. Phys. B555, 231 (1999).

[35] S. Cheng, Phys. Rev. D 99, 053005 (2019).

[36] J. F. Donoghue, J. Gasser, and H. Leutwyler, Nucl. Phys. B343, 341 (1990).

[37] M. Albaladejo and B. Moussallam, Eur. Phys. J. C 75, 488 (2015).

[38] W. F. Wang, J. Chai, and A. J. Ma, J. High Energy Phys. 03 (2020) 162.

[39] A. Abele, S. Bischoff, P. Blum, N. Djaoshvili, D. Engelhardt, A. Herbstrith, C. Holtzhaussen, M. Tischhauser, J. Adomeit, B. Kammle et al., Phys. Rev. D 57, 3860 (1998).

[40] S. M. Flatte, Phys. Lett. 63B, 224 (1976).

[41] H. Y. Cheng, C. K. Chua, K. C. Yang, and Z. Q. Zhang, Phys. Rev. D 87, 114001 (2013).

[42] P. A. Zyla et al. (Particle Data Group), Prog. Theor. Exp. Phys. 2020, 083 C01 (2020).

[43] V. M. Braun, D. Y. Ivanov, and G. P. Korchemsky, Phys. Rev. D 69, 034014 (2004).

[44] S. Aoki, Y. Aoki, D. Becirevic, C. Bernard, T. Blum, G. Colangelo, M. Della Morte, P. Dimopoulos, S. Dürr, H. Fukaya et al., Eur. Phys. J. C 77, 112 (2017).

[45] S. Cheng, A. Khodjamirian, and A. V. Rusov, Phys. Rev. D 102, 074022 (2020).

[46] S. S. Agaev, Phys. Rev. D 72, 074020 (2005).

[47] P. Ball and R. Zwicky, Phys. Lett. B 625, 225 (2005).

[48] G. Duplancic, A. Khodjamirian, T. Mannel, B. Melic, and N. Offen, J. High Energy Phys. 04 (2008) 014.

[49] A. Khodjamirian, T. Mannel, N. Offen, and Y. M. Wang, Phys. Rev. D 83, 094031 (2011).

[50] P. Ball, V. M. Braun, and A. Lenz, J. High Energy Phys. 05 (2006) 004.

[51] G. S. Bali, V. M. Braun, S. Bürger, M. Göckeler, M. Gruber, F. Hutzler, P. Korcyl, A. Schäfer, A. Sternbeck, and P. Wein (RQCD Collaboration), J. High Energy Phys. 08 (2019) 065 . 
[52] R. Arthur, P. A. Boyle, D. Brommel, M. A. Donnellan, J. M. Flynn, A. Juttner, T. D. Rae, and C. T. C. Sachrajda, Phys. Rev. D 83, 074505 (2011).

[53] A. Khodjamirian, T. Mannel, and M. Melcher, Phys. Rev. D 70, 094002 (2004).

[54] C. Shi, C. Chen, L. Chang, C. D. Roberts, S. M. Schmidt, and H. S. Zong, Phys. Rev. D 92, 014035 (2015).

[55] H. Leutwyler, Phys. Lett. B 378, 313 (1996).

[56] C. Amsler, Rev. Mod. Phys. 70, 1293 (1998).

[57] D. Parganlija and F. Giacosa, Eur. Phys. J. C 77, 450 (2017).
[58] G. Y. Wang, S. C. Xue, G. N. Li, E. Wang, and D. M. Li, Phys. Rev. D 97, 034030 (2018).

[59] H. Y. Cheng, C. K. Chua, and K. C. Yang, Phys. Rev. D 73, 014017 (2006).

[60] C. D. Lu, Y. M. Wang, and H. Zou, Phys. Rev. D 75, 056001 (2007).

[61] H. Y. Han, X. G. Wu, H. B. Fu, Q. L. Zhang, and T. Zhong, Eur. Phys. J. A 49, 78 (2013).

[62] Z. G. Wang, Eur. Phys. J. C 75, 50 (2015).

[63] Z. Q. Zhang and Z. J. Xiao, Chin. Phys. C 34, 528 (2010).

[64] Y. Li, Phys. Rev. D 89, 094007 (2014). 